\title{
THE FORMATION OF KILOPARSEC-SCALE H I HOLES IN DWARF GALAXIES*†
}

\author{
Steven R. Warren ${ }^{1}$, Daniel R. Weisz ${ }^{2}$, Evan D. Skillman ${ }^{1}$, John M. Cannon ${ }^{3}$, Julianne J. Dalcanton ${ }^{2}$, \\ Andrew E. Dolphin ${ }^{4}$, Robert C. Kennicutt, JR. ${ }^{5}$, BÄrbel Koribalski ${ }^{6}$, JÜrgen OtT $^{7}$, Adrienne M. STilP ${ }^{2}$, \\ SCHUYler D. VAN DYK ${ }^{8}$, Fabian WALTER ${ }^{9}$, AND ANDREW A. West ${ }^{10}$ \\ ${ }^{1}$ Department of Astronomy, University of Minnesota, 116 Church St. SE, Minneapolis, MN 55455, USA; warren@ astro.umn.edu, skillman@astro.umn.edu \\ ${ }^{2}$ Department of Astronomy, Box 351580, University of Washington, Seattle, WA 98195, USA; dweisz@ astro.washington.edu, jd@astro.washington.edu, \\ adrienne@astro.washington.edu \\ ${ }^{3}$ Department of Physics and Astronomy, Macalester College, 1600 Grand Avenue, St. Paul, MN 55125, USA; jcannon@ macalester.edu \\ ${ }^{4}$ Raytheon Company, 1151 East Hermans Road, Tucson, AZ 85756, USA; adolphin@ raytheon.com \\ ${ }^{5}$ Institute of Astronomy, University of Cambridge, Madingley Road, Cambridge CB3 0HA, UK; robk@ast.cam.ac.uk \\ ${ }^{6}$ Australia Telescope National Facility, CSIRO Astronomy and Space Science, P.O. Box 76, Epping, NSW 1710, Australia; Baerbel.Koribalski@ csiro.au \\ ${ }^{7}$ National Radio Astronomy Observatory, 520 Edgemont Road, Charlottesville, VA 22903, USA; jott@ nrao.edu \\ ${ }^{8}$ Spitzer Science Center/Caltech, Mailcode 220-6, Pasadena, CA 91125, USA; vandyk@ipac.caltech.edu \\ ${ }^{9}$ Max Planck Institut für Astronomie, Königstuhl 17, D-69117 Heidelberg, Germany; walter@mpia.de \\ ${ }^{10}$ Department of Astronomy, Boston University, 725 Commonwealth Avenue, Boston, MA 02215, USA; aawest@bu.edu \\ Received 2011 March 4; accepted 2011 May 20; published 2011 August 9
}

\begin{abstract}
The origin of kpc-scale holes in the atomic hydrogen ( $\mathrm{HI}$ ) distributions of some nearby dwarf irregular galaxies presents an intriguing problem. Star formation histories (SFHs) derived from resolved stars give us the unique opportunity to study past star-forming events that may have helped shape the currently visible $\mathrm{H}$ I distribution. Our sample of five nearby dwarf irregular galaxies spans over an order of magnitude in both total $\mathrm{H}$ I mass and absolute $B$-band magnitude and is at the low-mass end of previously studied systems. We use Very Large Array H I line data to estimate the energy required to create the centrally dominant hole in each galaxy. We compare this energy estimate to the past energy released by the underlying stellar populations computed from SFHs derived from data taken with the Hubble Space Telescope. The inferred integrated stellar energy released within the characteristic ages exceeds our energy estimates for creating the holes in all cases, assuming expected efficiencies. Therefore, it appears that stellar feedback provides sufficient energy to produce the observed holes. However, we find no obvious signature of single star-forming events responsible for the observed structures when comparing the global SFHs of each galaxy in our sample to each other or to those of dwarf irregular galaxies reported in the literature. We also fail to find evidence of a central star cluster in FUV or $\mathrm{H} \alpha$ imaging. We conclude that large $\mathrm{H}$ I holes are likely formed from multiple generations of star formation and only under suitable interstellar medium conditions.
\end{abstract}

Key words: galaxies: dwarf - galaxies: individual (DDO 181, Holmberg I, M81 Dwarf A, Sextans A, UGC 8508) - galaxies: ISM - ISM: structure

Online-only material: color figures

\section{INTRODUCTION}

Atomic hydrogen ( $\mathrm{H} \mathrm{I})$ observations of nearby galaxies reveal complex gas distributions. In many systems, the neutral interstellar medium (ISM) contains holes, shells, and/or cavities (e.g., Heiles 1979; Brinks \& Bajaja 1986; Puche et al. 1992; Oey \& Massey 1995; Kim et al. 1999; Walter \& Brinks 1999, 2001; Muller et al. 2003; Relaño et al. 2007; Chu 2008). Some holes in dwarf irregular (dIrr) galaxies are large enough that they become the dominant feature of the ISM, encompassing most, if not all of the optical disk (e.g., M81 Dwarf A: Sargent et al. 1983; Sagittarius DIG: Young \& Lo 1997; Holmberg I: Ott et al. 2001; DDO 88: Simpson et al. 2005; DDO 165: Cannon et al. 2011).

It has been suggested that these structures are created by feedback from stellar processes (e.g., stellar winds and supernovae (SNe) in OB associations; Weaver et al. 1977; Cash et al. 1980; McCray \& Kafatos 1987; Tenorio-Tagle \& Bodenheimer

\footnotetext{
* The Very Large Array telescope of the National Radio Astronomy Observatory is operated by Associated Universities, Inc. under a cooperative agreement with the National Science Foundation.

$\dagger$ Based on observations made with the NASA/ESA Hubble Space Telescope which is operated by the Association of Universities for Research in Astronomy, Inc., under NASA contract NAS 5-26555.
}

1988). Indeed, Ott et al. (2001), Simpson et al. (2005), Weisz et al. (2009), and Cannon et al. (2011) examined the stellar content within the $\mathrm{HI}$ holes of Holmberg I, DDO 88, Holmberg II, and DDO 165, respectively, and determined that the underlying stellar populations provide sufficient mechanical energy needed to create the observed holes within their estimated ages.

However, the premise that stellar winds and $\mathrm{SNe}$ are responsible for forming $\mathrm{HI}$ holes has been called into question. Heiles (1984) pointed out that the largest $(\sim 1 \mathrm{kpc})$ Galactic "supershells" seemed to require more energy than is available in a typical $\mathrm{OB}$ association. Looking at holes in the Large Magellanic Cloud, Kim et al. (1999) concluded that there is only a weak correlation between the locations of $\mathrm{H} \alpha$ emission and Hi holes. Perna \& Gaensler (2004) compared the locations of radio pulsars with Galactic holes and concluded that the largest holes are not consistent with the multiple $\mathrm{SNe}$ from a single-aged cluster formation scenario. Similarly, Hatzidimitriou et al. (2005) compared the locations of known $\mathrm{H}$ I holes in the Small Magellanic Cloud to OB associations, supergiants, Cepheids, Wolf-Rayet stars, SN remnants, and star clusters, and found that there are $\sim 1.5$ times as many holes without evidence for recent star formation as there are with 
recent star formation tracers. Further, the largest H I shell in the Local Group dIrr IC 1613 contains 27 OB associations in projection (Borissova et al. 2004; Silich et al. 2006), but the inferred stellar input energy does not account for the estimated hole creation energy (Silich et al. 2006). Taken together, these findings argue against a single-aged cluster being responsible for forming large $\mathrm{H}$ i holes; however, stellar processes from multiple generations of star formation remains viable.

Other authors have proposed alternative formation hypotheses to the SNe origin. Efremov et al. (1998) and Loeb \& Perna (1998) postulate that a high-energy gamma-ray burst (GRB) from the death of a single massive star could create kpc-sized holes in the ISM, thus offering an explanation for holes without a detectable underlying cluster. These authors assume the energy from GRBs is emitted isotropically. However, GRBs release most of their energy in bidirectional beams (Rhoads 1997), making this scenario less likely to produce large $\mathrm{H}$ i holes. Other models of $\mathrm{H}$ I hole formation without $\mathrm{SNe}$ are gravitational and thermal instabilities (Wada et al. 2000; Dib \& Burkert 2005), a fractal ISM (Elmegreen 1997), H I dissolution by UV radiation (Vorobyov \& Shchekinov 2004), and ram pressure stripping (Bureau \& Carignan 2002).

Yet another alternative formation model was suggested by Tenorio-Tagle (1981), who investigated the effect of high velocity cloud (HVC) collisions with the gas disks of galaxies, concluding that the amount of energy deposited into the ISM can be of the same order as an $\mathrm{SN}\left(\sim 10^{51} \mathrm{erg}\right)$ or more. One observational prediction of this model is a half-circle arc seen in an $\mathrm{HI}$ position-velocity (P-V) diagram. The half-circle arc arises from the gas being pushed to one direction, corresponding to the direction of travel of the HVC. Some observational support for this idea is reported by Heiles $(1979,1984)$ who point out that the most energetic Galactic shells in their study all have half-circle arc signatures in $\mathrm{P}-\mathrm{V}$ space.

It is likely that to a certain degree most of the above physical processes must play a role in the formation of at least some Hi holes. To determine which process is the most crucial, Vorobyov \& Basu (2005) carried out numerical simulations. They investigated three of the above $\mathrm{H}$ i hole formation scenarios (multiple $\mathrm{SNe}$, a single GRB, and an HVC collision) by simulating the ISM structure of the dIrr galaxy Holmberg I. They showed that multiple $\mathrm{SNe}$ explosions more accurately reproduce the Hi morphology of Holmberg I than the GRB or HVC models.

The study of Rhode et al. (1999) raised doubt about the stellar feedback from a single-aged cluster idea. These authors investigated the numerous H i holes of Holmberg II with Very Large Array (VLA) $\mathrm{H}$, ground-based $B V R$, and narrowband $\mathrm{H} \alpha$ images. They expected to find the remnants of the OB associations (A, F, and $\mathrm{G}$ type main-sequence stars) left behind in the holes, but instead discovered that the integrated light was inconsistent with the required cluster masses needed for a multiple SNe formation scenario from a single-aged cluster. However, Stewart $\&$ Walter (2000) found that the most prominent hole in IC 2574 is coincident with a prominent stellar cluster. Recently, Weisz et al. (2009) used deep Hubble Space Telescope (HST) photometry to resolve some of the stellar population of Holmberg II and showed that all of the holes observed with HST contain evidence of multiple stellar generations and are consistent with the holes being formed from the energy input into the ISM by these past stars. Their study brought to light the need to reconstruct the star formation histories (SFHs; and thus the available stellar input energy) from the resolved stars using the methodology developed by Dolphin (2002). These conclusions have been reinforced by the Cannon et al. (2011) investigation of a large ( $\sim 775$ pc) Hi hole in DDO 165. These authors compared the energy budget of the underlying stellar population versus time to estimated hole creation energies. They conclude that the stars have produced more than enough energy to create the observed Hi hole.

Evidence is mounting that large $\mathrm{H}_{\mathrm{I}}$ holes are not formed by a single-aged stellar cluster but by multiple generations of star formation working in concert. In this study we investigate dwarf galaxies in which centrally dominant $\mathrm{H}$ i holes have been identified in order to address the multiple stellar generation hypothesis: DDO 181 (UGC 8651), Holmberg I (UGC 5139, DDO 63, KDG 57; Ott et al. 2001), M81 Dwarf A (KDG 52; Sargent et al. 1983), Sextans A (UGCA 205, DDO 75; Skillman et al. 1988), and UGC 8508. Studying the specific problem of large holes in dIrr galaxies has some advantages over studies of larger spiral galaxies. For example, dIrr galaxies rotate as solid bodies for the bulk of their $\mathrm{HI}$ disks (e.g., Skillman 1996), limiting the effects of destructive shearing forces due to differential rotation. These forces can shorten the lifetimes of the holes in spiral arms of larger galaxies. Also, solid body rotation allows the young stars to remain very near their birth places on timescales of $\sim 10^{8}$ years (for observational support of this see Dohm-Palmer et al. 2002 and for a statistical approach see Bastian et al. 2011). Given the above, we can use deep HST imaging of regions within the radii of the holes to create spatially resolved SFHs. From the SFHs we calculate a cumulative stellar energy budget (i.e., stellar input energy over time). We compare the input stellar energy to the energy required to create the holes derived from VLA H I images. This comparison gives us a handle on the ability of stellar feedback to create large holes in the neutral ISM of dIrr galaxies. We analyze the SFHs for these five galaxies and compare them to SFHs of dIrr galaxies without central $\mathrm{HI}_{\mathrm{I}}$ holes to determine if any obvious hole creation signature is seen. Finally, we search for centralized clusters responsible for forming the hole in each galaxy with FUV and $\mathrm{H} \alpha$ imaging.

\section{DATA PRODUCTS}

\subsection{H I Data}

H I data were taken from two large atomic hydrogen surveys: The Hi Nearby Galaxy Survey (THINGS; AW0605; Walter et al. 2008) and the VLA Advanced Camera for Surveys (ACS) Nearby Galaxy Survey Treasury (VLA-ANGST; AO0215; Ott et al. 2008). Observations were made using the VLA in B, C, and D array configurations. Reduction of the THINGS data has been described in detail by Walter et al. (2008). Standard AIPS spectral line reduction procedures were followed. Flux, bandpass, and phase calibration were performed using VLA calibrators. The final moment 0 maps were flux corrected (Jörsäter \& van Moorsel 1995) and produced images with a natural weighted resolution of $\sim 14^{\prime \prime}=\sim 260 \mathrm{pc}$ for Holmberg I and $\sim 15^{\prime \prime}=\sim 260 \mathrm{pc}$ for M81 Dwarf A.

Reduction of the VLA-ANGST data is described in an upcoming paper, but closely followed the procedures of the THINGS pipeline. Deviations from the THINGS reduction pipeline were only required where problems arose due to the addition of Extended VLA (EVLA) antennas into the array. Baselines between EVLA antennas were affected by power aliased into the first $0.5 \mathrm{MHz}$ of the baseband in the conversion 

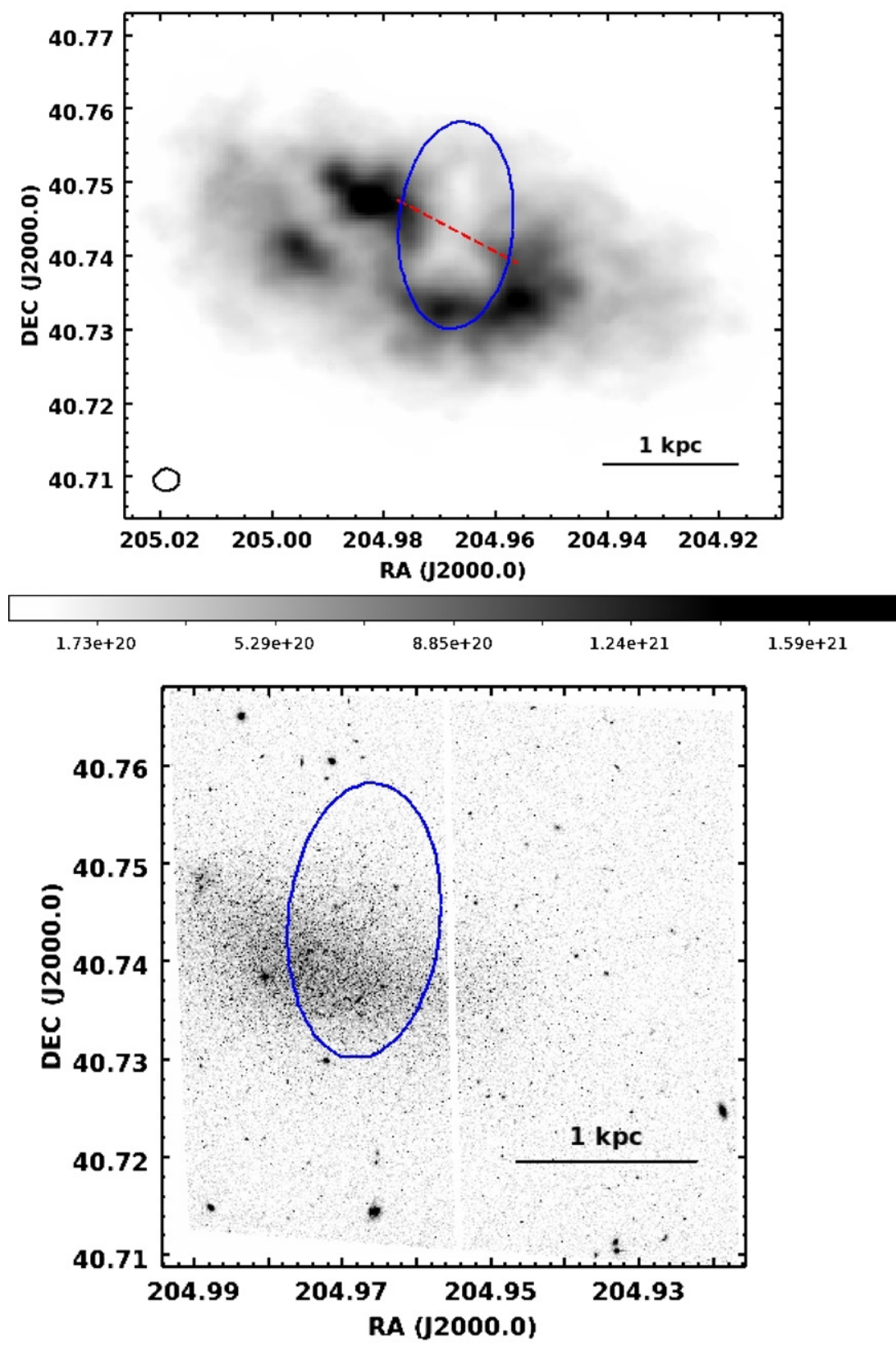

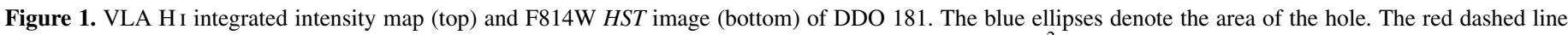

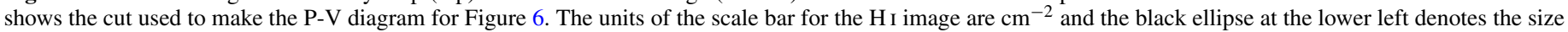
and shape of the synthesized beam.

(A color version of this figure is available in the online journal.)

of the signal from digital to analog. These baselines were subsequently removed from the data. The baselines between VLA and EVLA antennas were unaffected and remain in the data. The final moment 0 maps were flux corrected (Jörsäter \& van Moorsel 1995) and produced images with a natural weighted resolution of $\sim 12^{\prime \prime}=\sim 175 \mathrm{pc}$ for DDO $181, \sim 11^{\prime \prime}=\sim 75 \mathrm{pc}$ for Sextans A, and $\sim 13^{\prime \prime}=\sim 165$ pc for UGC 8508 . We show the $\mathrm{H}$ I integrated intensity maps for the sample galaxies in the top panels of Figures $1-5$. Table 1 gives the relevant $\mathrm{H}$ i image properties for each galaxy and Table 2 gives the general observed properties of each galaxy in our sample.

\subsection{HST Data}

HST/ACS and WFPC2 imaging were obtained as part of three HST programs. Data for Sextans A were first described in
Table 1

Beam and Resolution of $\mathrm{H}$ I Images

\begin{tabular}{lcccc}
\hline \hline Name & $\begin{array}{c}B_{\text {maj }} \\
\left({ }^{\prime \prime}\right)\end{array}$ & $\begin{array}{c}B_{\min } \\
\left({ }^{\prime \prime}\right)\end{array}$ & $\begin{array}{c}\text { Beam Position Angle } \\
\left({ }^{\circ}\right)\end{array}$ & $\begin{array}{c}\text { Velocity Resolution } \\
\left(\mathrm{km} \mathrm{s}^{-1}\right)\end{array}$ \\
\hline DDO 181 & 12.5 & 10.5 & -80.4 & 0.63 \\
Holmberg I & 14.7 & 12.7 & -41.6 & 2.5 \\
M81 Dwarf A & 15.9 & 14.2 & 10.2 & 2.5 \\
Sextans A & 11.6 & 10.9 & 47.6 & 0.63 \\
UGC 8508 & 14.0 & 11.5 & 88.1 & 0.63 \\
\hline
\end{tabular}

Dohm-Palmer et al. (1997, 2002) and were reprocessed by Holtzman et al. (2006). Imaging of Holmberg I and M81 Dwarf A were obtained as part of a larger HST program aimed at studying M81 Group dwarf galaxies (GO-10605; PI: Skillman; 

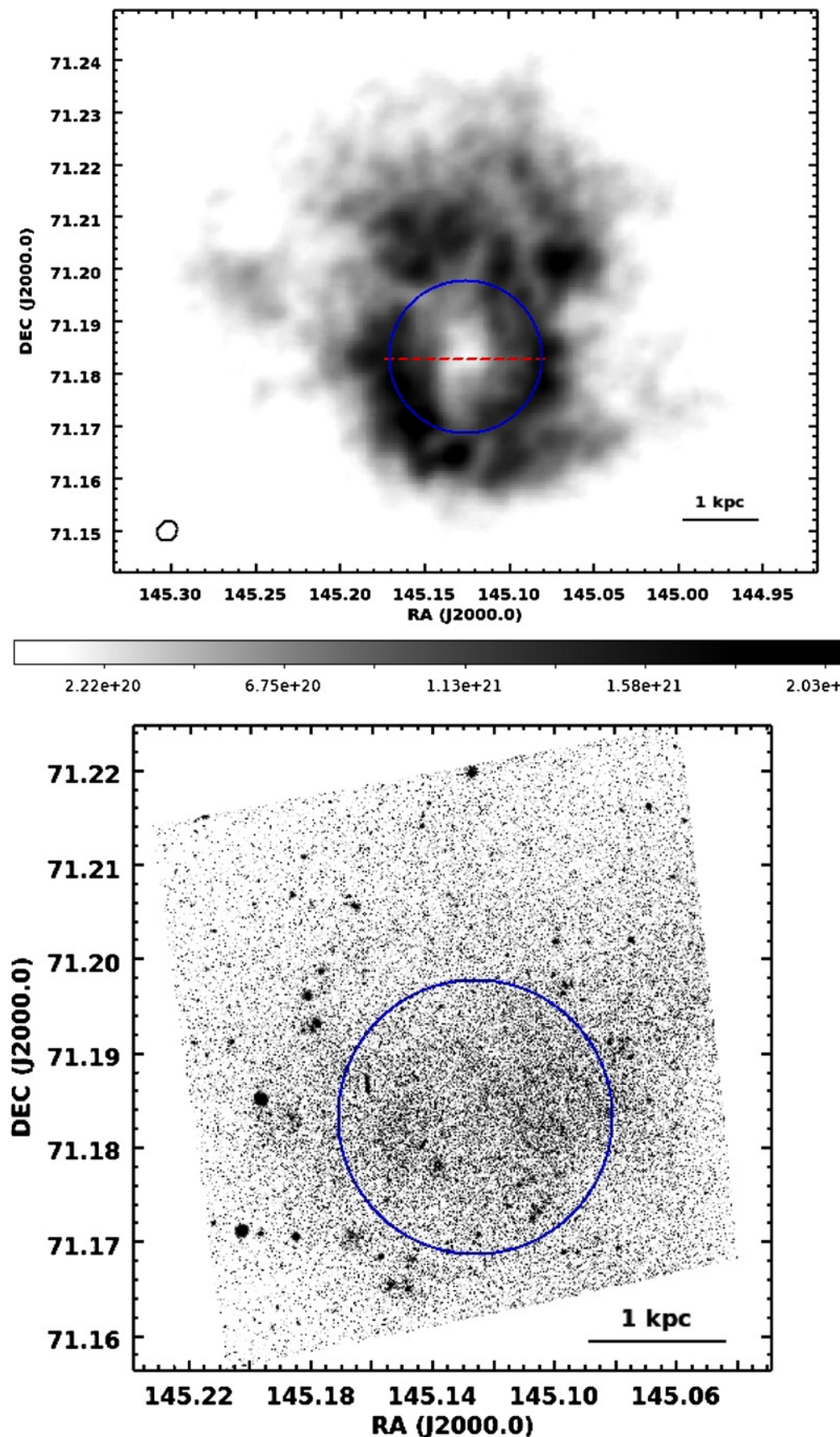

Figure 2. VLA H I integrated intensity map (top) and F814W HST image (bottom) of Holmberg I. The blue circles denote the area of the hole. The red dashed line shows the cut used to make the P-V diagram for Figure 6. The units of the scale bar for the H I image are $\mathrm{cm}^{-2}$ and the black ellipse at the lower left denotes the size and shape of the synthesized beam.

(A color version of this figure is available in the online journal.)

Weisz et al. 2008) and were reprocessed along with DDO 181 and UGC 8508 within the ACS Nearby Galaxy Survey Treasury (ANGST) project (Dalcanton et al. 2009). Here, we briefly summarize the details of the photometry and measurements of the SFHs. Full details of the photometry are listed in Holtzman et al. (2006) and Dalcanton et al. (2009), while details of the SFHs are listed in Weisz et al. (2008, 2011). Following standard data reduction with the HST pipeline, photometry for WFPC2 data was performed using HSTphot (Dolphin 2000) while the ACS data were processed with the ACS-specific module of DOLPHOT (Dolphin 2000). The raw photometry was then filtered to exclude non-stellar point-spread functions, and the resultant photometric catalogs range from a low of
17,450 stars for M81 Dwarf A to 121,198 stars for Holmberg I. Approximately 500,000 artificial star tests were performed for each galaxy to compute the completeness functions and quantify uncertainties due to observational effects. The bottom panels of Figures $1-5$ show the F814W images of each galaxy.

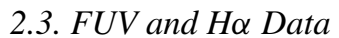

We utilize FUV and $\mathrm{H} \alpha$ imaging obtained through the $11 \mathrm{Mpc} \mathrm{H} \alpha$ UV Galaxy Survey (11HUGS; Kennicutt et al. 2008). Full details of the observations and calibrations can be found in Kennicutt et al. (2008) and Lee et al. (2011). Briefly, standard GALEX calibration and data processing were used 

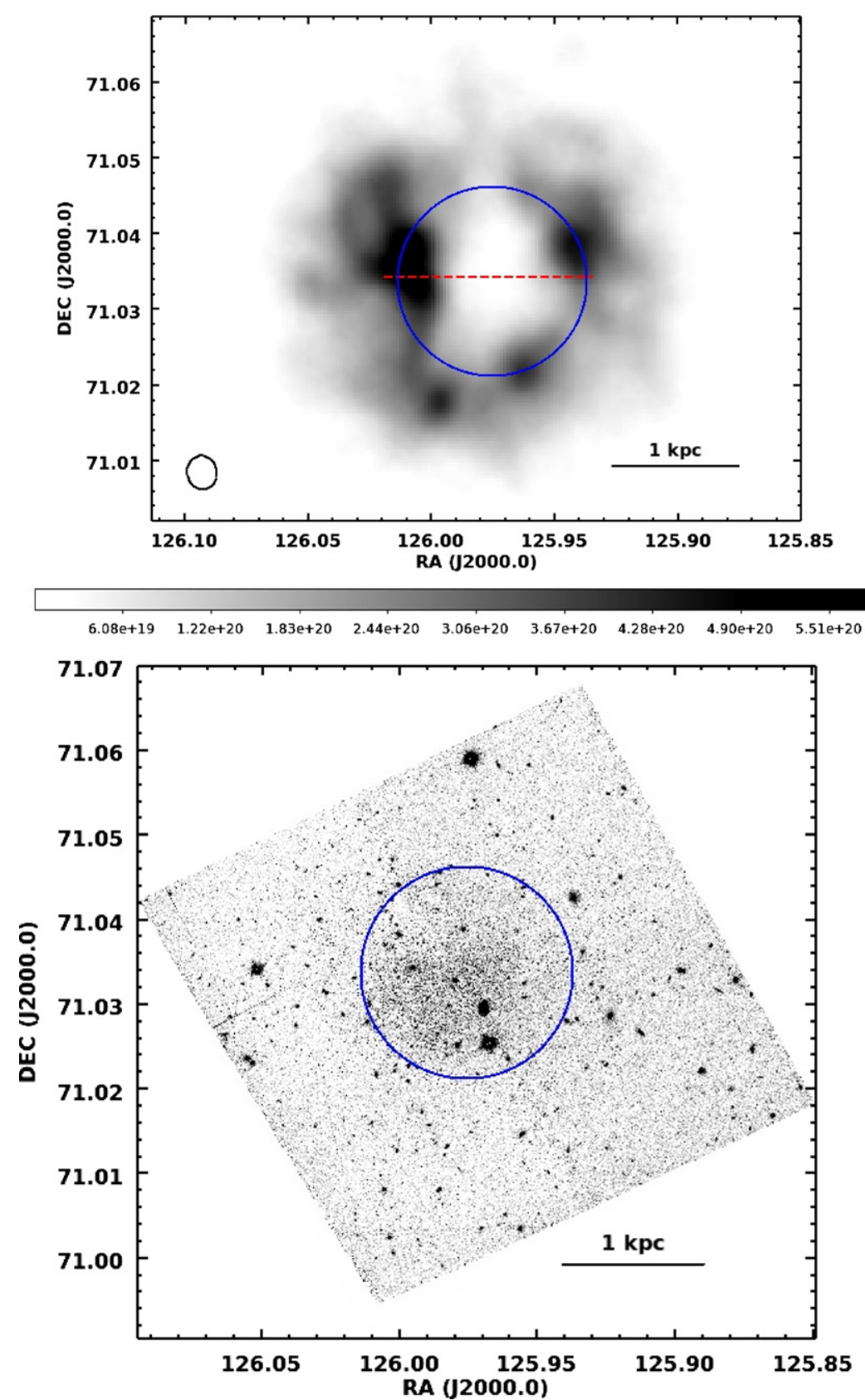

Figure 3. VLA H I integrated intensity map (top) and F814W HST image (bottom) of M81 Dwarf A. The blue circles denote the area of the hole. The red dashed line shows the cut used to make the P-V diagram for Figure 6. The units of the scale bar for the $\mathrm{H}$ I image are $\mathrm{cm}^{-2}$ and the black ellipse at the lower left denotes the size and shape of the synthesized beam.

(A color version of this figure is available in the online journal.)

following procedures outlined in Morrissey et al. (2007). The GALEX UV images were initially cleaned of foreground stars and background galaxies before photometry was performed. Background levels were estimated from pixels beyond the $B$-band $25 \mathrm{mag} \operatorname{arcsec}^{-1}$ isophote where UV galaxy flux is assumed to be zero. The background flux is required to estimate the asymptotic magnitude of the galaxy. Azimuthally averaged surface brightness profiles were made and a cumulative magnitude versus cumulative-magnitude gradient was fit by a line, where the $y$-intercept is determined to be the asymptotic magnitude of the galaxy.

$\mathrm{H} \alpha$ imaging was obtained from three different telescopes between 2001 and 2005: the Steward Observatory Bok $2.3 \mathrm{~m}$ telescope on Kitt Peak, the Lennon 1.8 m Vatican Advanced
Technology Telescope (VATT) on Mt. Graham, AZ, and the $0.9 \mathrm{~m}$ telescope at the Cerro Tololo Inter-American Observatory (CTIO). Common IRAF reduction procedures were followed. $R$-band imaging was scaled to the continuum level of narrowband imaging containing $\mathrm{H} \alpha$ and $\left[\mathrm{N}_{\mathrm{II}}\right] \lambda \lambda 6548,84$ emission lines. The $\mathrm{H} \alpha+[\mathrm{N}$ II $]$ images were then continuum subtracted to isolate the emission lines. Finally, images were flux calibrated using spectrophotometric standard stars.

\section{HOLE DEFINITIONS}

Holes in the H I distribution of dIrr galaxies come in a variety of shapes and sizes. Brinks \& Bajaja (1986) defined three different types of holes depending on their characteristics in a 


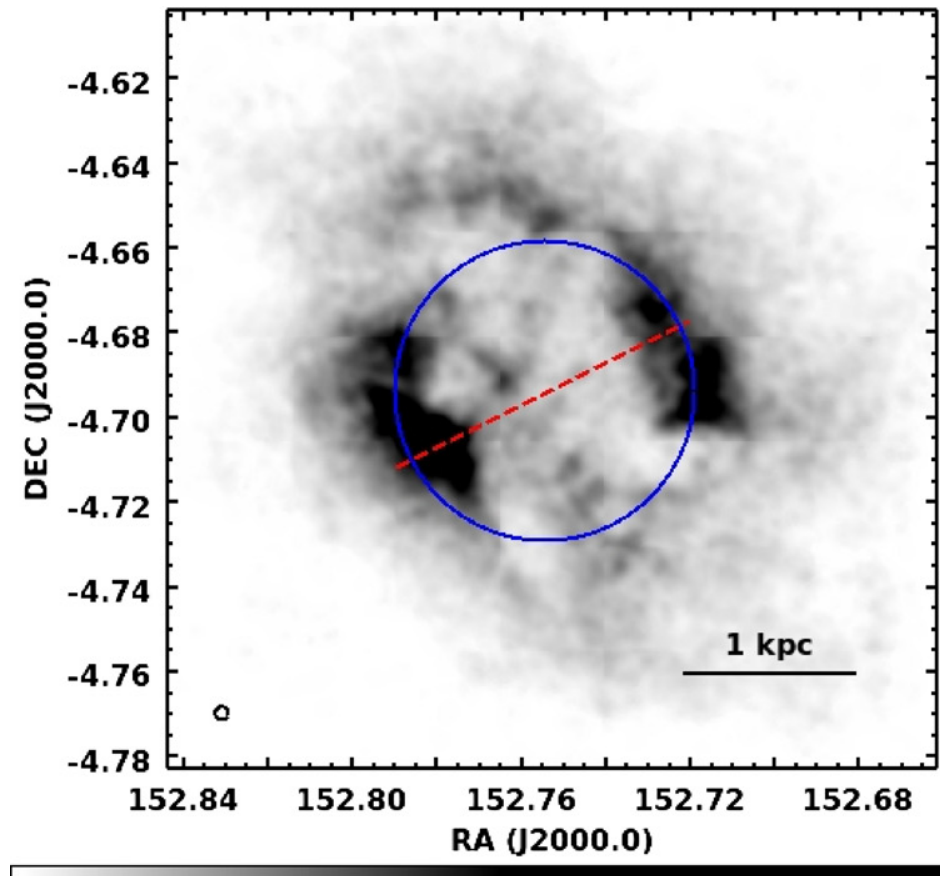

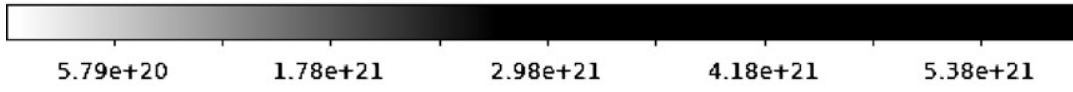

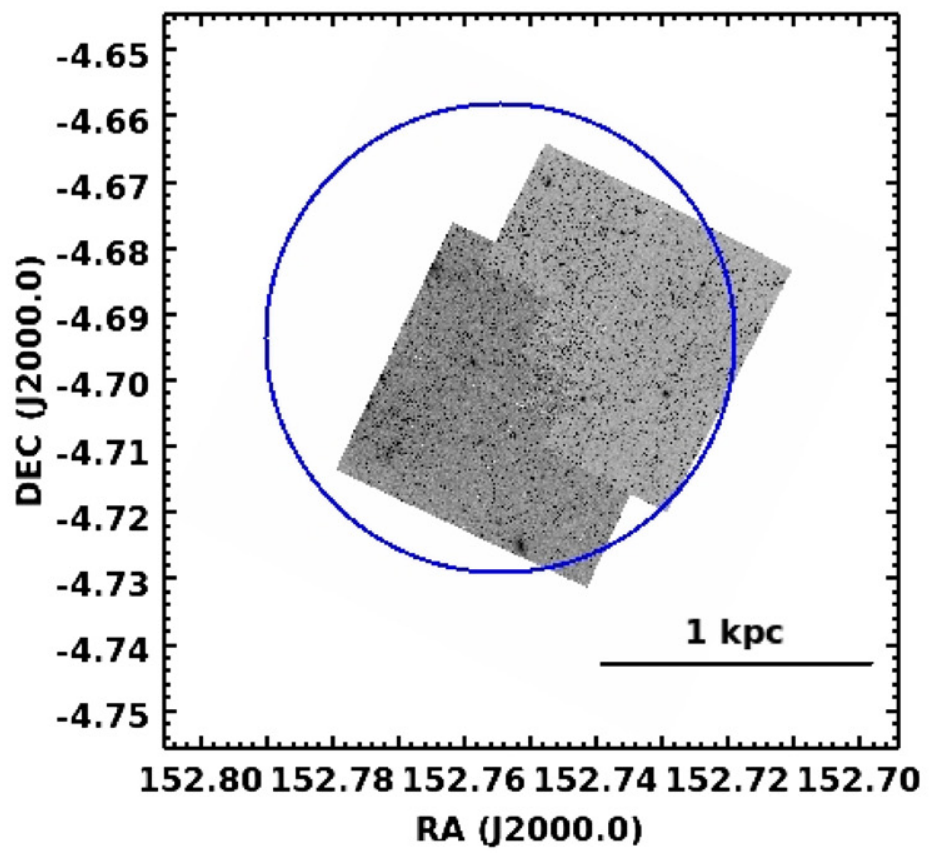

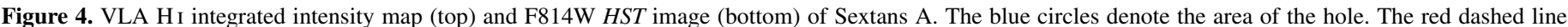

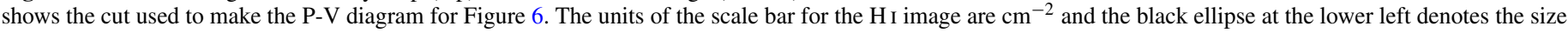
and shape of the synthesized beam.

(A color version of this figure is available in the online journal.)

$\mathrm{P}-\mathrm{V}$ diagram. A Type 1 hole is a structure that has expanded out of the disk (i.e., blow-out) and shows a discontinuous profile in a $\mathrm{P}-\mathrm{V}$ diagram. Type 1 holes are among the most commonly observed as they are readily visible as depressions in $\mathrm{H}$ I integrated intensity maps. Type 2 holes are those offset from the plane of the host galaxy such that only one side has undergone blow-out. These holes have a half-circle arc signature in a $\mathrm{P}-\mathrm{V}$ diagram similar to what might be observed from an HVC collision (e.g., Vorobyov \& Basu 2005). Finally, a Type 3 hole is a "classic" hole where a coherent expanding structure can be seen in a $\mathrm{P}-\mathrm{V}$ or radius-velocity diagram. This hole type allows for its current expansion velocity to be measured directly. Figure 6 shows a representative P-V diagram of each galaxy. All of the centrally dominant holes in this study are Type 1 holes, that is, their $\mathrm{P}-\mathrm{V}$ diagrams are discontinuous and show no signs of expansion discernible from their average velocity dispersions. We are therefore unable to define expansion velocities for the holes and instead use the average velocity dispersion as a proxy for this value; we discuss this further in Section 4.1. 

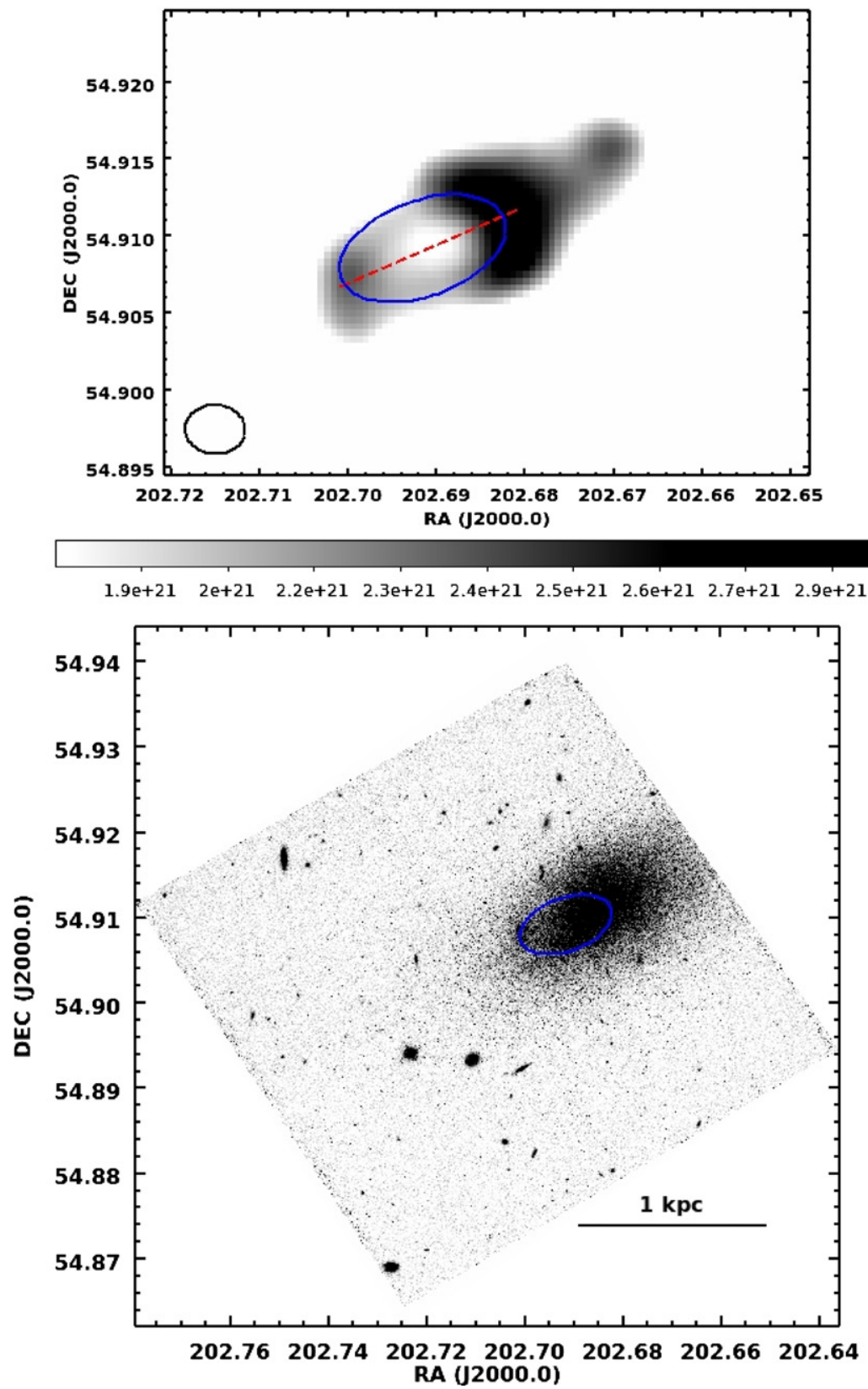

Figure 5. VLA HI integrated intensity map (top) and F814W HST image (bottom) of UGC 8508. The blue ellipses denote the area of the hole. The red dashed line shows the cut used to make the P-V diagram for Figure 6. The units of the scale bar for the $\mathrm{H} \mathrm{I}$ image are $\mathrm{cm}^{-2}$ and the black ellipse at the lower left denotes the size and shape of the synthesized beam.

(A color version of this figure is available in the online journal.)

Table 2

General Galaxy Properties

\begin{tabular}{|c|c|c|c|c|c|c|c|c|c|c|c|}
\hline Galaxy & $\begin{array}{c}\text { R.A. } \\
(\mathrm{J} 2000.0)\end{array}$ & $\begin{array}{c}\text { Decl. } \\
(\mathrm{J} 2000.0)\end{array}$ & $\begin{array}{c}d \\
(\mathrm{Mpc})\end{array}$ & $\begin{array}{l}\text { Scale } \\
\left(\mathrm{pc} /^{\prime \prime}\right)\end{array}$ & $m_{B}{ }^{\mathrm{a}}$ & $M_{B}$ & $\begin{array}{c}L_{B} \\
\left(10^{7} L_{\odot}\right)\end{array}$ & $\begin{array}{c}M_{\mathrm{HI}_{\mathrm{I}}} \\
\left(10^{7} M_{\odot}\right)\end{array}$ & $\begin{array}{c}N_{\mathrm{H}, \text { Peak }} \\
\left(10^{21} \mathrm{~cm}^{-2}\right)\end{array}$ & $\begin{array}{c}N_{\mathrm{H}, \mathrm{Ave}} \\
\left(10^{20} \mathrm{~cm}^{-2}\right)\end{array}$ & $\begin{array}{c}\left\langle\sigma_{v}\right\rangle \\
\left(\mathrm{km} \mathrm{s}^{-1}\right)\end{array}$ \\
\hline DDO 181 & $13^{\mathrm{h}} 39^{\mathrm{m}} 53^{\mathrm{s}} .8$ & $+40^{\circ} 44^{\prime} 21^{\prime \prime}$ & 3.1 & 15.0 & 14.4 & -13.0 & 1.12 & 2.60 & 1.77 & 3.12 & 8.2 \\
\hline Holmberg I & $09^{\mathrm{h}} 40^{\mathrm{m}} 32^{\mathrm{s}} \cdot 3$ & $+71^{\circ} 10^{\prime} 56^{\prime \prime}$ & 3.9 & 18.9 & 13.4 & -14.5 & 4.45 & 14.6 & 2.26 & 2.78 & 7.9 \\
\hline M81 Dwarf A & $08^{\mathrm{h}} 23^{\mathrm{m}} 55^{\mathrm{s}} .1$ & $+71^{\circ} 01^{\prime} 56^{\prime \prime}$ & 3.4 & 16.5 & 16.3 & -11.4 & 0.26 & 1.07 & 0.61 & 1.09 & 7.0 \\
\hline Sextans A & $10^{\mathrm{h}} 11^{\mathrm{m}} 00^{\mathrm{s}} .8$ & $-04^{\circ} 41^{\prime} 34^{\prime \prime}$ & 1.4 & 6.79 & 11.7 & -14.0 & 2.81 & 6.80 & 5.98 & 5.16 & 9.8 \\
\hline UGC 8508 & $13^{\mathrm{h}} 30^{\mathrm{m}} 44^{\mathrm{s}} .4$ & $+54^{\circ} 54^{\prime} 36^{\prime \prime}$ & 2.6 & 12.6 & 14.0 & -13.1 & 1.22 & 2.20 & 2.98 & 2.42 & 10.3 \\
\hline
\end{tabular}

Notes. Columns are galaxy name, right ascension (R.A.), declination (decl.), distance (d), image scale, apparent $B$-band magnitude ( $\left.m_{B}\right)$, absolute $B$-band magnitude $\left(M_{B}\right), B$-band luminosity $\left(L_{B}\right)$, H I mass $\left(M_{\mathrm{H}}\right)$, peak and average $\mathrm{H}_{\mathrm{I}}$ column density $\left(N_{\mathrm{H}}\right.$, Peak,$N_{\mathrm{H}}$, Ave $)$, and the average velocity dispersion measured in the second moment map $\left(\left\langle\sigma_{v}\right\rangle\right)$.

${ }^{a}$ Karachentsev et al. (2004), apparent magnitudes are corrected for Galactic foreground extinction. 

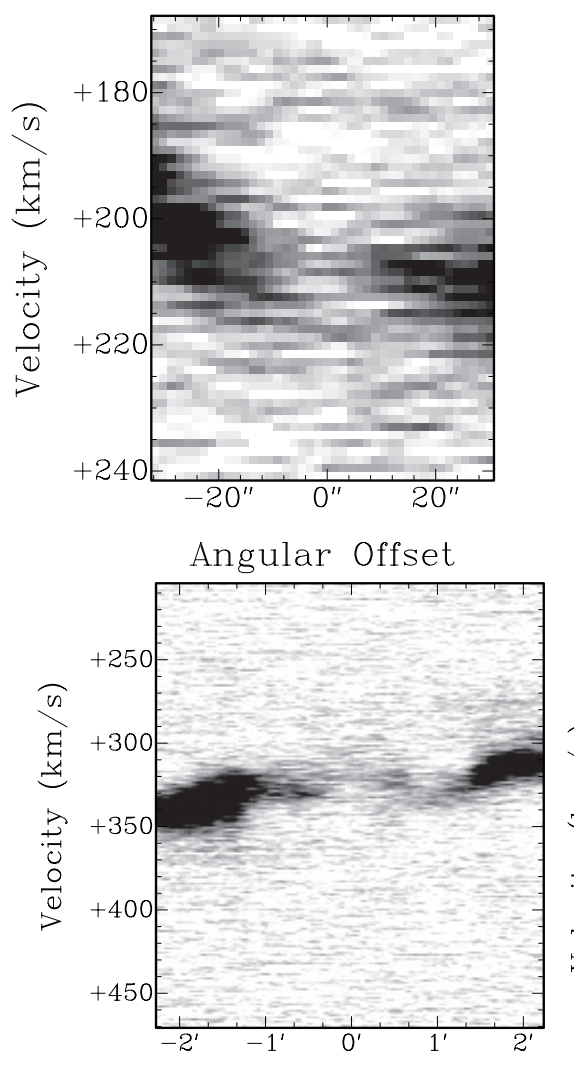

Angular Offset

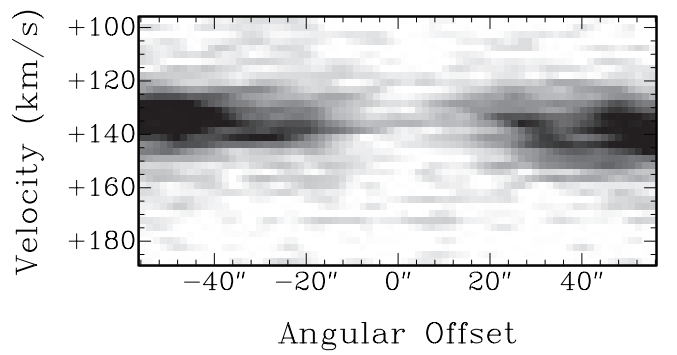

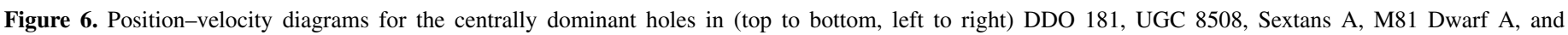

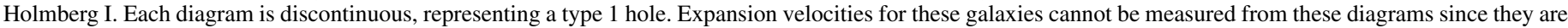
indistinguishable from the average velocity dispersion in the galaxy. The gray scale reflects the $\mathrm{H}_{\mathrm{I}}$ intensity.

We define the locations and shapes of the holes visually from the $\mathrm{H}$ i integrated intensity maps. We assume circular shapes for Holmberg I, M81 Dwarf A, and Sextans A, but define elliptical shapes for the holes in DDO 181 and UGC 8508. Defining holes visually from the integrated intensity map is straightforward for the large structures in our sample of galaxies. Recent work by Bagetakos et al. (2011) also identified holes visually on a sample of 20 THINGS galaxies, although they also used $\mathrm{P}-\mathrm{V}$ diagrams in order to identify smaller, less obvious holes. Automated search algorithms designed to detect $\mathrm{H}_{\mathrm{I}}$ holes do exist (e.g., Thilker et al. 1998; Mashchenko et al. 1999; Daigle et al. 2003; Ehlerová \& Palouš 2005). These routines are used mainly to find holes not readily seen in the integrated intensity maps but within the spectral data cubes. Since our holes are all easily identifiable in the integrated intensity maps (Figures 1-5) these algorithms are unnecessary for this work.

The large hole identified in each galaxy is a single structure except for that of Sextans A. Sextans A has a complex H I distribution consisting of a general depression surrounded by
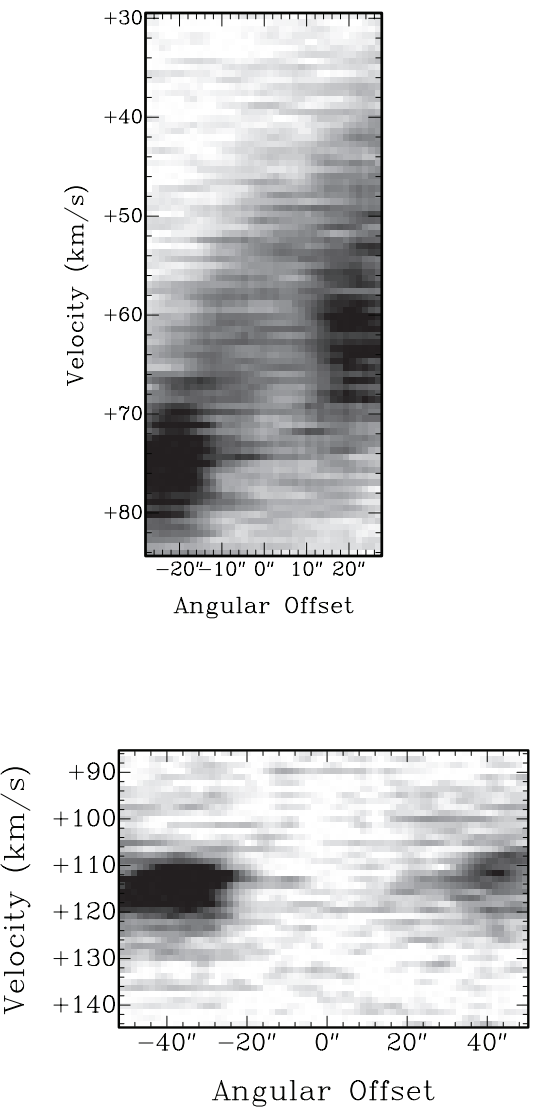

two larger, higher column density clouds. The central depression seems to be filled with multiple holes of various sizes and shapes (Puche \& Westpfahl 1994). However, previous authors, based on lower angular resolution data (e.g., Skillman et al. 1988; van Dyk et al. 1998) have described this feature as a single hole. This gives us a unique opportunity to test the effect of resolution on our observed holes. The holes we observe in the more distant galaxies of our sample may also be filled with numerous, smaller holes that we are not sensitive to. If we describe the Hi morphology of Sextans A as a single, large hole the amount of energy we derive for its creation will be larger than required to form many smaller holes. So if it is shown that the underlying stars have ample energy to create one large hole, then certainly they could produce many smaller holes; thus, we include it here and treat it as a single, central hole.

The radial extents of the holes were defined by plotting the average $\mathrm{H}$ i column density versus radius as was done previously by Ott et al. (2001) and Simpson et al. (2005). This procedure was done with the IRAF task ELLIPSE which computes the 


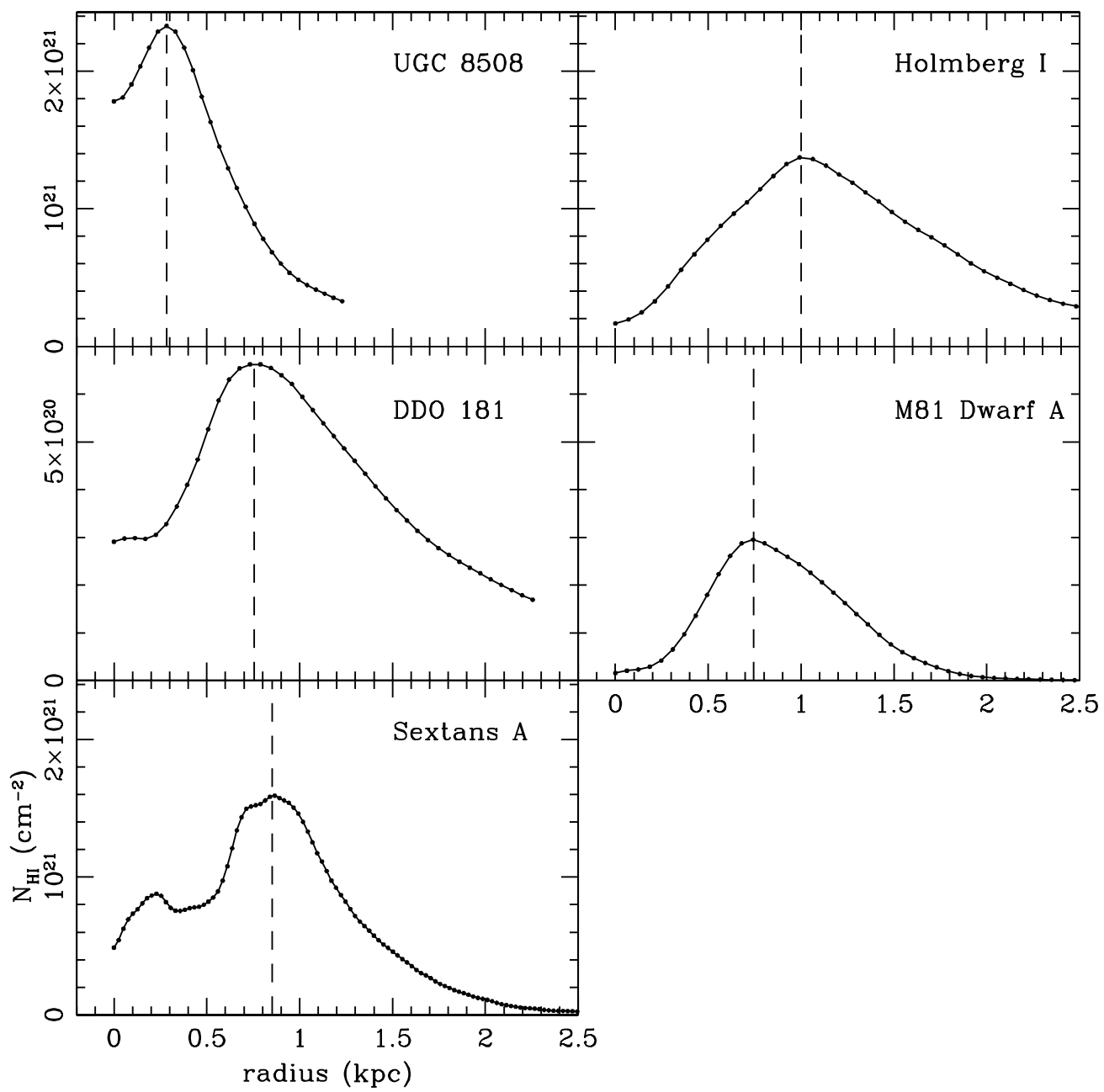

Figure 7. Azimuthally averaged column density vs. radius for each galaxy. The vertical dashed lines denote the adopted radii of the holes.

Table 3

Hole Properties

\begin{tabular}{|c|c|c|c|c|c|c|c|c|c|c|c|}
\hline Galaxy & $\begin{array}{l}\text { R.A. } \\
\text { (J2000) }\end{array}$ & $\begin{array}{l}\text { Decl. } \\
\text { (J2000) }\end{array}$ & $\begin{array}{l}r_{\text {hole }} \\
(\mathrm{pc})\end{array}$ & $\begin{array}{c}\text { P.A. } \\
\left({ }^{\circ}\right)\end{array}$ & $\begin{array}{c}\tau_{\text {char }} \\
(\mathrm{Myr})\end{array}$ & $\begin{array}{l}E_{\text {stars }} \\
\text { (erg) }\end{array}$ & $\begin{array}{l}E_{\max } \\
\text { (erg) }\end{array}$ & $\begin{array}{l}E_{\min } \\
(\mathrm{erg})\end{array}$ & $\epsilon_{\max }$ & $\epsilon_{\min }$ & $\begin{array}{l}\text { age }_{\text {alt }} \\
(\mathrm{Myr})\end{array}$ \\
\hline DDO 181 & $13^{\mathrm{h}} 39^{\mathrm{m}} 52^{\mathrm{s}} .1$ & $+40^{\circ} 44^{\prime} 39^{\prime \prime} 0$ & 755 (415) & 85 & 90 & $1.1 \times 10^{54}$ & $5.3 \times 10^{53}$ & $3.9 \times 10^{52}$ & $48 \%$ & $3.5 \%$ & $>500,240$ \\
\hline Holmberg I & $09^{\mathrm{h}} 40^{\mathrm{m}} 30^{\mathrm{s}}$ & $+71^{\circ} 11^{\prime} 01^{\prime \prime} 8$ & 1000 & 0 & 124 & $3.4 \times 10^{54}$ & $1.2 \times 10^{54}$ & $6.7 \times 10^{52}$ & $35 \%$ & $2.0 \%$ & 395,170 \\
\hline M81 Dwarf A & $08^{\mathrm{h}} 23^{\mathrm{m}} 54^{\mathrm{s}} 1$ & $+71^{\circ} 02^{\prime} 01^{\prime \prime} .5$ & 745 & 0 & 104 & $1.4 \times 10^{54}$ & $5.0 \times 10^{53}$ & $3.5 \times 10^{51}$ & $35 \%$ & $0.3 \%$ & $>500,390$ \\
\hline Sextans A & $10^{\mathrm{h}} 11^{\mathrm{m}} 01.1$ & $-04^{\circ} 41^{\prime} 37^{\prime \prime} .0$ & 850 & 0 & 85 & $2.2 \times 10^{55}$ & $6.4 \times 10^{53}$ & $1.1 \times 10^{53}$ & $3 \%$ & $0.5 \%$ & 27,19 \\
\hline UGC 8508 & $13^{\mathrm{h}} 30^{\mathrm{m}} 45^{\mathrm{s}} .9$ & $+54^{\circ} 54^{\prime} 33^{\prime \prime} .0$ & 285 (157) & 20 & 27 & $1.8 \times 10^{53}$ & $2.2 \times 10^{52}$ & $9.2 \times 10^{50}$ & $12 \%$ & $0.5 \%$ & 30,20 \\
\hline
\end{tabular}

Notes. Columns are galaxy name, right ascension (R.A.), and declination (decl.) of the center of the hole, the hole radius (semi-major axis length for DDO 181 and UGC 8508; semi-minor axis length is in parentheses), the position angle of the hole (P.A.), the characteristic age of the hole ( $\left.\tau_{\text {char }}\right)$, the cumulative stellar energy budget at the characteristic age of the hole $\left(E_{\mathrm{stars}}\right)$, the upper limit energy for creating the hole derived from Equation (1) ( $E_{\max }$ is derived assuming $\left.N_{\mathrm{H}_{\mathrm{I}}}=10^{21} \mathrm{~cm}^{-2}\right)$, the lower energy limit to create the hole $\left(E_{\mathrm{min}}\right.$ is derived using the average observed $\left.N_{\mathrm{H}_{\mathrm{I}}}\right)$, the upper and lower stellar feedback efficiencies $\left(\epsilon_{\max }\right.$ and $\left.\epsilon_{\min }\right)$, and alternative ages (age ${ }_{a l t}$ are the age estimates for stellar feedback efficiencies of $10 \%$ and $20 \%$ using the upper limits of the hole creation energies; see Section 5).

average value on the boundary of an input ellipse incrementally from the starting location outward in predefined step sizes. The hole radius is then defined as the peak in this radial profile. If a hole is created in a uniformly dense medium, the hole boundary will be defined as the location of the shock front and will be identified as a delta function in a similar radial profile. Once the hole stops expanding, the shock front will broaden and disperse over time. We do not find any evidence for a shock front, but if the gas were being piled up our assumption of treating the peak of this profile as the radius of the hole is reasonable. Figure 7 shows the radius versus average column density profiles. For the elliptical holes in DDO 181 and UGC 8508 the radius is defined as the semi-major axis length. Each profile rises to a maximum before declining again, except for the H I profile of Sextans A. The radial profile for Sextans A shows substructure before the global peak. This substructure is due to the non-uniform nature of the central depression mentioned above, including a region of higher column density gas clumped just offset from the defined hole center. The holes range in radius from $\sim 285$ pc (UGC 8508) to $\sim 970 \mathrm{pc}$ (Holmberg I). We plot the hole boundary with a blue circle or ellipse on the H I and optical images (Figures 1-5) as well as list their individual properties in Table 3. 


\section{ENERGETICS}

We use a blast wave model to estimate the total amount of energy needed to make a cavity in the ISM. We then compare this to an estimate of the stellar feedback energy derived from the resolved stellar population to test the idea that the energy from stellar feedback is the dominant source in shaping the neutral ISM in these galaxies. To make this comparison we determine three things: (1) the amount of energy that was required to form the $\mathrm{HI}$ holes, (2) the amount of available energy from stellar feedback, and (3) the relevant timescale for energy injection. A timescale in which to compare these two calculations is not so straightforward and is described below.

\subsection{Timescales}

We must first determine the relevant timescale over which the input stellar energy shaped the hole. One such timescale is the kinematic age of the hole, defined as the ratio of the radius of the hole by the expansion velocity. Looking at different cuts in a $\mathrm{P}-\mathrm{V}$ diagram we find no evidence for current expansion in any of these holes. This behavior is expected for holes that have blown out of the disk as the input energy will preferentially follow the path of least resistance (along the blow-out direction). Further expansion will quickly stall out and become indistinguishable from the velocity dispersion of the background gas distribution (e.g., Vorobyov et al. 2004).

Since there is no evidence that the holes are currently expanding, we use the average velocity dispersion of the gas as a proxy for the expansion velocity. We define the average velocity dispersion as the mean value of the second moment map over the entire galaxy. For holes that have not blown out of the disk, using this value will provide an upper limit to the true age of the hole, because it assumes the expansion rate was constant throughout the evolution, which certainly was not true during the initial formation stages. However, since all of our holes are of Type 1, the kinematic age can be thought of as a characteristic age $\left(\tau_{\text {char }}\right)$, not as an upper or lower limit, since we cannot know for sure how long the structure has maintained its current configuration. Our computed characteristic ages are all roughly $100 \mathrm{Myr}$ (see Table 3) with the exception of UGC 8508 which has an age $~ 30$ Myr. These ages fall in the range of reported hole ages in other galaxies of $\sim 10^{6}-10^{8}$ yr (e.g., Oey \& Massey 1995; Walter \& Brinks 1999; Hatzidimitriou et al. 2005; Weisz et al. 2009; Bagetakos et al. 2011).

\subsection{Energy Required to Form H I Holes}

In 1974, Chevalier derived an empirical relation between

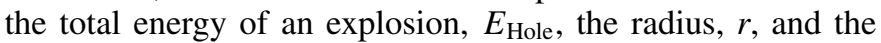
expansion velocity, $v$, of the cavity left behind, taking into account the initial volume density, $n_{o}$, of the medium:

$$
E_{\text {Hole }}=5.3 \times 10^{43}\left(\frac{n_{o}}{\mathrm{~cm}^{-3}}\right)^{1.12}\left(\frac{r}{\mathrm{pc}}\right)^{3.12}\left(\frac{v}{\mathrm{~km} \mathrm{~s}^{-1}}\right)^{1.40} \mathrm{erg} .
$$

This equation has been used by many authors to estimate the energy required to make $\mathrm{H}$ i holes (e.g., Ott et al. 2001; Simpson et al. 2005; Weisz et al. 2009). However, one must keep in mind the many assumptions that go into using Equation (1). First, Equation (1) assumes a homogeneous medium throughout the entire evolution of the blast wave. For smaller holes that have not blown out of the disk, this assumption provides an upper limit to the required energy, since a realistic ISM density profile is stratified, allowing the shock wave to propagate with less resistance and grow to larger sizes. In the scenario of larger holes that have blown out of the disk, Equation (1) just gives us an estimate of the needed energy, but cannot be interpreted as an upper or lower limit. Second, defining the initial volume density for the cavity is non-trivial. One needs to know the thickness (column density) and distribution (e.g., exponential, Gaussian, etc.) of the gas to accurately define the volume density at any given point of an H I map. Further complicating matters, $n_{o}$ is the initial gas volume density and since there already exists an evacuated cavity, this number can only be estimated. The uncertainty in the calculated energy value may be as high as an order of magnitude and possibly more depending on the true initial conditions. For example, if one assumes a value for $n_{o}$ of 0.01 whereas the "true" value should be 0.1 , the derived energy value will be off by $\sim 8 \%$, assuming the radius and expansion velocity used are correct.

To address these issues, we have decided to follow the method of Ott et al. (2001) to estimate a lower limit to the volume density. For this we assume that the gas follows a Gaussian distribution from the midplane, which leads to

$$
N_{\mathrm{HI}}=\sqrt{2 \pi} h n_{o},
$$

where $N_{\mathrm{HI}}$ is the H I column density, $h$ is the gas scale height, and $n_{o}$ is the midplane gas volume density. Thus, in principle, a measurement of $h$ leads to an estimate of $n_{o}$. Defining the gas scale height, however, is not straightforward.

There have been many ideas put forth as to how one would correctly estimate the gas scale height from the observables of face-on galaxies (e.g., van der Kruit 1981; Padoan et al. 2001; Elmegreen et al. 2001; Weisz et al. 2009). Weisz et al. (2009) and Cannon et al. (2011) used a method described in Oh et al. (2008) to define the scale height as a function of radius. This process involves defining the stellar mass surface density of the galactic disk from Spitzer $3.6 \mu \mathrm{m}$ imaging, and then assuming that the stars are the only significant source of surface density. For galaxies with baryonic masses greater than $\sim 10^{9} M_{\odot}$, the stars in the disk should dominate the local gravitational potential (see Mac Low \& Ferrara 1999 and references therein). However, for less massive galaxies, the dark matter plays an increasing role in the gravitational potential and the gas scale height will be smaller than that derived from the stellar distribution alone. Banerjee et al. (2011) recently modeled the effects of dark matter, gas self-gravity, and the stellar content of four dwarf galaxies, including Holmberg II, on the H I vertical scale height. Their derived scale height as a function of radius is generally smaller than the one derived by Weisz et al. (2009). The effect is more pronounced at larger radii. Unfortunately, we do not have the correct inputs to follow the method of Banerjee et al. (2011), thus for uniformity we follow the steps of Ott et al. (2001) who used the gas scale height equation derived by van der Kruit (1981) to relate the gas scale height to the average gas velocity dispersion, $\sigma_{\text {gas }}$, and the disk total mass density, $\rho_{\mathrm{t}}$ :

$$
h(R)=\frac{\sigma_{\mathrm{gas}}}{\sqrt{4 \pi G \rho_{\mathrm{t}}}} .
$$

The gas velocity dispersion should be affected by the underlying dark matter potential as well as the stellar mass distribution. 
Ott et al. (2001) combined Equations (2) and (3) yielding

$$
\begin{aligned}
h & =\left(\sqrt{8 \pi} G m_{p}\right)^{-1} \frac{\sigma_{\mathrm{gas}}^{2}}{\left(\rho_{\mathrm{t}} / \rho_{\mathrm{H}}\right) N_{\mathrm{H}_{\mathrm{I}}}} \\
& =579\left(\frac{\sigma_{\mathrm{gas}}}{10 \mathrm{~km} \mathrm{~s}^{-1}}\right)^{2}\left(\frac{N_{\mathrm{H}_{\mathrm{I}}}}{10^{21} \mathrm{~cm}^{-2}}\right)^{-1}\left(\frac{\rho_{\mathrm{H}}}{\rho_{\mathrm{t}}}\right) \mathrm{pc},
\end{aligned}
$$

where $G$ is the gravitational constant, $m_{p}$ is the proton mass, and $\rho_{\mathrm{H}}$ is the H mass density. Similarly to Ott et al. (2001), we assume that $\left(\rho_{\mathrm{HI}} / \rho_{\mathrm{t}}\right)=\left(M_{\mathrm{HI}} / M_{\mathrm{t}}\right)$ with $M_{\mathrm{t}}=M_{\mathrm{HI}}+M_{\mathrm{He}}+$ $M_{\text {stars }}, M_{\mathrm{He}}=0.3 M_{\mathrm{HI}}$, and a stellar mass-to-light ratio ( $\left.M_{\mathrm{stars}}\right)$ $\left.L_{B}\right)=1$. We then use the $M_{\mathrm{HI}}, L_{B}$, and the average $N_{\mathrm{H} \text { I }}$ values given in Table 2 to derive $\left(\rho_{\mathrm{HI}_{\mathrm{I}}} / \rho_{\mathrm{t}}\right), h$, and midplane $n_{o}$. Finally, we calculate lower limits to the input energies $\left(E_{\min }\right)$ required to create the hole in the $\mathrm{H}$ I distribution by substituting Equations (2) and (4) into Equation (1).

Skillman et al. (1987) find that massive stars form around H I column density peaks of $\gtrsim 10^{21} \mathrm{~cm}^{-2}$. Therefore, we can derive upper limits to the required input energy $\left(E_{\max }\right)$ for hole formation by assuming this $N_{\mathrm{H}}$ value for each galaxy. This new energy value represents an upper limit because using $N_{\mathrm{H} \text { I }}=10^{21} \mathrm{~cm}^{-2}$ assumes the entire ISM within the radius of the hole was at this value prior to forming the hole. This was not the case for each of the galaxies in our sample, with the possible exception of UGC 8508. The column density of the gas within and around the hole in UGC 8508 is on the order of $10^{21} \mathrm{~cm}^{-2}$; thus, the resulting energy estimate may be closer to the expected value than it is for the holes in the other galaxies. The new $N_{\mathrm{H}_{\mathrm{I}}}$ value leads to new estimates of $n_{o}$ using Equations (4) and (2). Equation (1) then leads us to upper limits to the amount of hole formation energy. Table 3 gives the estimated lower and upper limits required to form the holes for each galaxy.

An alternative method of calculating hole formation energies to the Chevalier (1974) model was proposed by McCray \& Kafatos (1987) and implemented recently by Chakraborti \& Ray (2011). The McCray \& Kafatos (1987) model disregards the single stellar explosion model in favor of continuous energy injection. They propose that the number of $\mathrm{SNe}$ required to make the hole is given by

$$
\left(N_{*} E_{51} / n_{0}\right)=\left(R_{S} / 97 \mathrm{pc}\right)^{2}\left(V_{S} / 5.7 \mathrm{~km} \mathrm{~s}^{-1}\right)^{3},
$$

where $N_{*}$ is the number of SNe, $E_{51}$ is the energy per $\mathrm{SN}$ in units of $10^{51} \mathrm{erg}, n_{0}$ is the initial volume density of the gas, $R_{S}$ is the radius of the hole, and $V_{S}$ is the current expansion velocity of the hole. This equation provides values slightly lower than those given by Equation (1). For example, using the radius and velocity dispersion defined above for DDO 181 in place of $R_{S}$ and $V_{S}$, respectively, Equation (5) gives us $105 \mathrm{SNe}=$ $1.05 \times 10^{53} \mathrm{erg}$. This value is lower than the $5.3 \times 10^{53} \mathrm{erg}$ that Equation (1) provides us with. The larger values provided by Equation (1) give us more confidence that we are truly deriving upper limits for the amount of energy required to make these large holes. The lower limits that we derive using Equation (1) are also slightly higher than what Equation (5) would provide for the same reasons as the above example. However, if the amount of available energy from the stars (see Section 4.4) far exceeds our upper limit derived using Equation (1), the value used for our lower limit is less important. Our estimates for the amount of energy needed to create the observed $\mathrm{H}$ i holes are in the ranges derived by other authors for similar holes in other galaxies (e.g., Ott et al. 2001; Simpson et al. 2005; Cannon et al. 2011).

\subsection{Individual Calculations of H I Hole Energetics}

\subsection{1. $D D O 181$}

DDO 181 was first listed in the dwarf galaxy catalog of van den Bergh (1959). Using the tip of the red giant branch, Dalcanton et al. (2009) estimate a distance to DDO 181 of $3.1 \mathrm{Mpc}$. At this distance, the linear size of the $\mathrm{H}_{\mathrm{I}}$ disk has a maximum of $\sim 4.4 \mathrm{kpc}$. DDO 181 has an integrated H I flux density of $11.5 \mathrm{Jy} \mathrm{km} \mathrm{s}^{-1}$ corresponding to a total H I mass of $2.6 \times 10^{7} M_{\odot}$.

The hole in DDO 181 is readily visible in the H I integrated intensity map (Figure 1) as a peanut-shaped depression near the center of the H I disk. To determine the size of the Hi hole we plot the average $\mathrm{H}$ I column density versus radius (Figure 7). We defined the center and ellipticity of the hole in DDO 181 visually from the $\mathrm{HI}_{\mathrm{I}}$ integrated intensity maps, placing the center of the hole at $\alpha=13^{\mathrm{h}} 39^{\mathrm{m}} 52^{\mathrm{s}} \cdot 1, \delta=+40^{\circ} 44^{\prime} 39^{\prime}$. 0 . Following the procedure outlined in Section 3 we derive a radius of $740 \mathrm{pc}$. Using Equation (1) we derive a range of hole creation energies of $3.9 \times 10^{52}$ to $5.3 \times 10^{53} \mathrm{erg}$.

\subsubsection{Holmberg I}

Holmberg I is a dIrr galaxy discovered by Holmberg (1950) in a photometric study of nearby galaxies. Dalcanton et al. (2009) fit the tip of the red giant branch to determine a distance to Holmberg I of $3.9 \mathrm{Mpc}$. Its distance and apparent $B$-band magnitude $\left(m_{B}\right)$ of 13.4 give it an absolute $B$-band magnitude $\left(M_{B}\right)$ of -14.5 , placing it among the least luminous observed dIrr galaxies (Begum et al. 2008). The $\mathrm{H}_{\mathrm{I}}$ integrated flux density of $40.1 \mathrm{Jy} \mathrm{km} \mathrm{s}^{-1}$ corresponds to a total H I mass for Holmberg I of $1.4 \times 10^{8} M_{\odot}$. Figure 2 is the $\mathrm{H}_{\mathrm{I}}$ integrated intensity map which shows the large hole in the ISM, roughly $45^{\prime \prime}$ South of the dynamical center of the galaxy (Ott et al. 2001). The H i hole also dominates the optical disk of the galaxy (Figure 2). We present the observed characteristics of Holmberg I in Table 2.

We perform the same radial analysis as above to characterize the size of the hole. We plot the azimuthally averaged $\mathrm{H}$ i column density versus radius in Figure 7. The peak in this distribution is at $\sim 53^{\prime \prime}$ or a radius of $1000 \mathrm{pc}$. Ott et al. (2001) derive a hole radius of $\sim 52^{\prime \prime}$ but used a distance of $3.6 \mathrm{Mpc}$, giving the hole a radius of $850 \mathrm{pc}$. Also, the VLA data have been reprocessed by the THINGS (Walter et al. 2008) reduction pipeline, changing some of the observed parameters. For example, Ott et al. (2001) observed an average velocity dispersion of roughly $9.0 \mathrm{~km} \mathrm{~s}^{-1}$ whereas we measure $7.9 \mathrm{~km} \mathrm{~s}^{-1}$. Despite these differences, their hole creation value $\left(E_{\text {hole }} \lesssim 1.2 \times 10^{53} \mathrm{erg}\right)$ falls within the range of values we derive for Holmberg I of $6.7 \times 10^{52}$ to $1.2 \times 10^{54} \mathrm{erg}$. On the other hand, Bagetakos et al. (2011) used the $\mathrm{P}-\mathrm{V}$ diagram instead of the column density radial profile we use to derive the size of this same hole. They derive a very discrepant hole radius of only $67.5 \mathrm{pc}$ and thus a hole creation value of $2.4 \times 10^{50} \mathrm{erg}$.

A check of the plausibility of our hole creation values comes from the numerical study of Vorobyov et al. (2004). They simulated the H I morphology of Holmberg I with a two-dimensional hydrodynamics code (ZEUS-2D) and injected different energies over $30 \mathrm{Myr}$ to approximate multiple SNe from a single age cluster of varying masses. The model that best matched the observed morphology of Holmberg I injected the energy of $\sim 300 \mathrm{SNe}$ of $10^{51}$ erg each or a total input energy of $3 \times 10^{53} \mathrm{erg}$. This value falls within our estimated hole creation energies from above. 

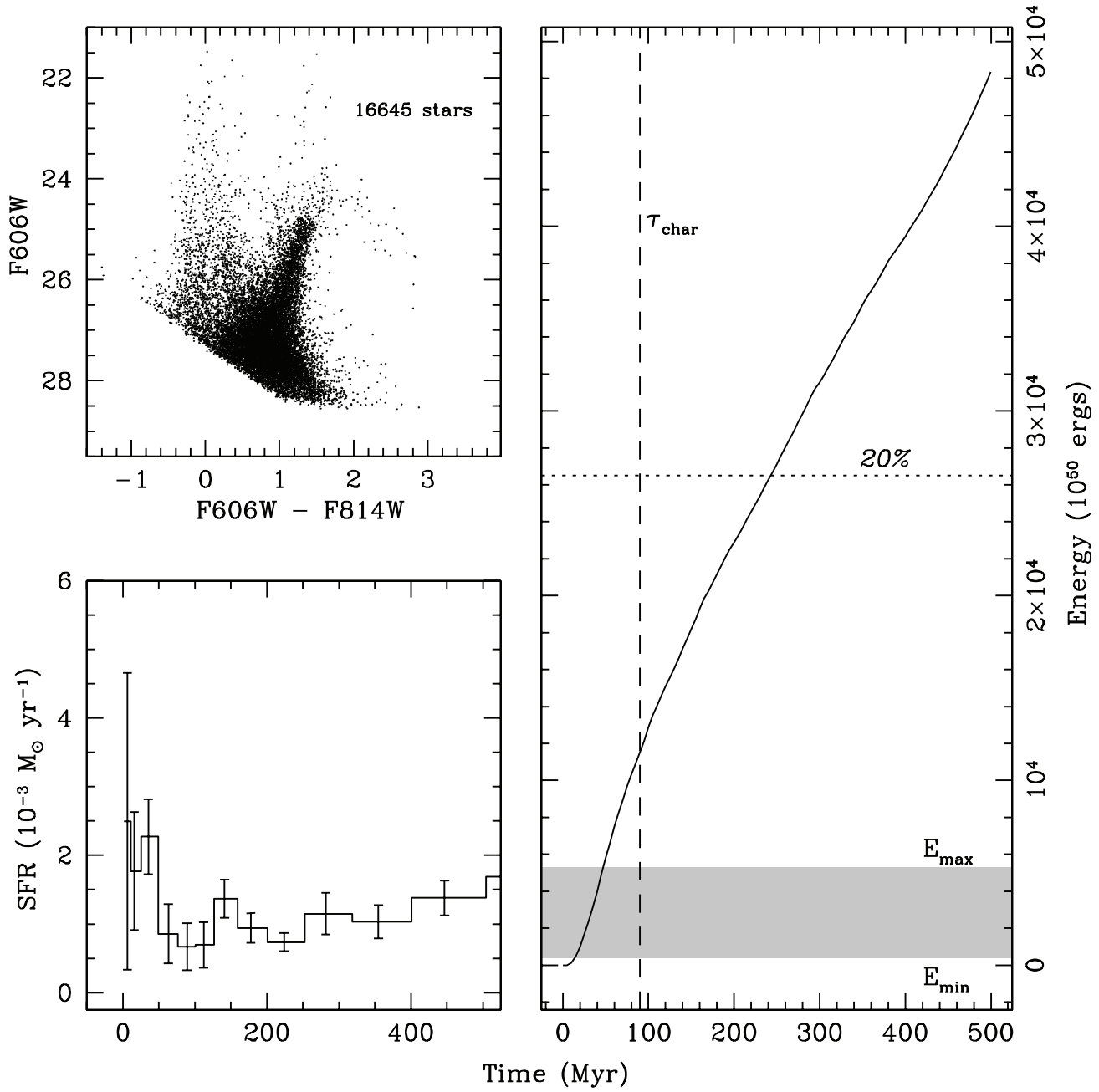

Figure 8. DDO 181-Upper left: the (F606W, F606W-F814W) CMD for the stars within the radius of the central H I hole. Lower left: the SFH over the past 500 Myr for the stars within the central H I hole. Right: the cumulative energy due to stellar winds and SNe calculated by using the SFH as input into STARBURST99 (Leitherer et al. 1999). The shaded region denotes the range in energy needed to create the H I hole from Section 4.2. The vertical, dashed line is the characteristic age of the hole. The horizontal dashed lines represent the $10 \%$ and/or $20 \%$ feedback efficiency levels using the upper limit of hole creation energy.

\subsubsection{M81 Dwarf A}

M81 Dwarf A was first listed in a survey of Sculptor-type dwarf galaxies by Karachentseva (1968). Eleven years later, Lo \& Sargent (1979) rediscovered M81 Dwarf A in an atomic hydrogen search around Holmberg II in the M81 group of galaxies. Recent estimates place M81 Dwarf A at a distance of 3.4 Mpc (Dalcanton et al. 2009). This sets the observed angular size of the $\mathrm{H}$ I disk of $\sim 220^{\prime \prime}$ equal to a linear diameter of $\sim 3.6 \mathrm{kpc}$. The integrated H I flux density of $4.1 \mathrm{Jy} \mathrm{km} \mathrm{s}^{-1}$ implies a total H I mass of $1.1 \times 10^{7} M_{\odot}$. M81 Dwarf A was the least massive galaxy observed in the THINGS sample and is near the lower mass end of observed dIrr galaxies. Table 2 lists the relevant properties of M81 Dwarf A.

The detailed study of Sargent et al. (1983) investigated the overall Hi morphology of M81 Dwarf A, pointing out the dominant ring feature seen in the ISM. Figure 3 shows the VLA H I integrated intensity map of M81 Dwarf A. This image reveals the large hole seen in the ISM which encompasses most of the optical disk (see Figure 3). The peak in the average $\mathrm{HI}_{\mathrm{I}}$ column density versus radius (Figure 7) corresponds to a radius of $\sim 45^{\prime \prime}$ or $\sim 745 \mathrm{pc}$. From this radius and properties listed in Table 2 we derive a range of hole creation energies of $3.5 \times$ $10^{51}$ to $5.0 \times 10^{53} \mathrm{erg}$.

\subsubsection{Sextans A}

Sextans A is a relatively close galaxy at a distance of $1.4 \mathrm{Mpc}$ (Dalcanton et al. 2009). Given its distance, it has also been extensively studied. Skillman et al. (1988) studied the H I distribution of Sextans A noting the ring-like central depression. Puche \& Westpfahl (1994) described this depression as "twisted and sheared." These authors also point out that the majority of the optical disk is contained within the central depression (see Figure 4). Dohm-Palmer et al. $(1997,2002)$ used HST observations of the resolved stars to derive an SFH of Sextans A, noting that the most recent star formation is occurring near the peaks in the H I. van Dyk et al. (1998) used ground-based observations to confirm these results and went on to show that the older stars (50-100 Myr old) are more centrally concentrated than the youngest stars. This seems to follow a propagating star formation hypothesis (Gerola \& Seiden 1978) suggested to occur in an SN and stellar-wind-driven hole expansion (Puche \& Westpfahl 1994). This pattern, however, is not seen in the HST data of Dohm-Palmer et al. (2002).

Although the central depression is comprised of multiple holes in our observation as discussed in Section 3, for uniformity we treat it here as one large hole. We define the center of the hole to be located at $\alpha=10^{\mathrm{h}} 11^{\mathrm{m}} 01^{\mathrm{s}} .1, \delta=-04^{\circ} 41^{\prime} 37^{\prime \prime} .0$, just 


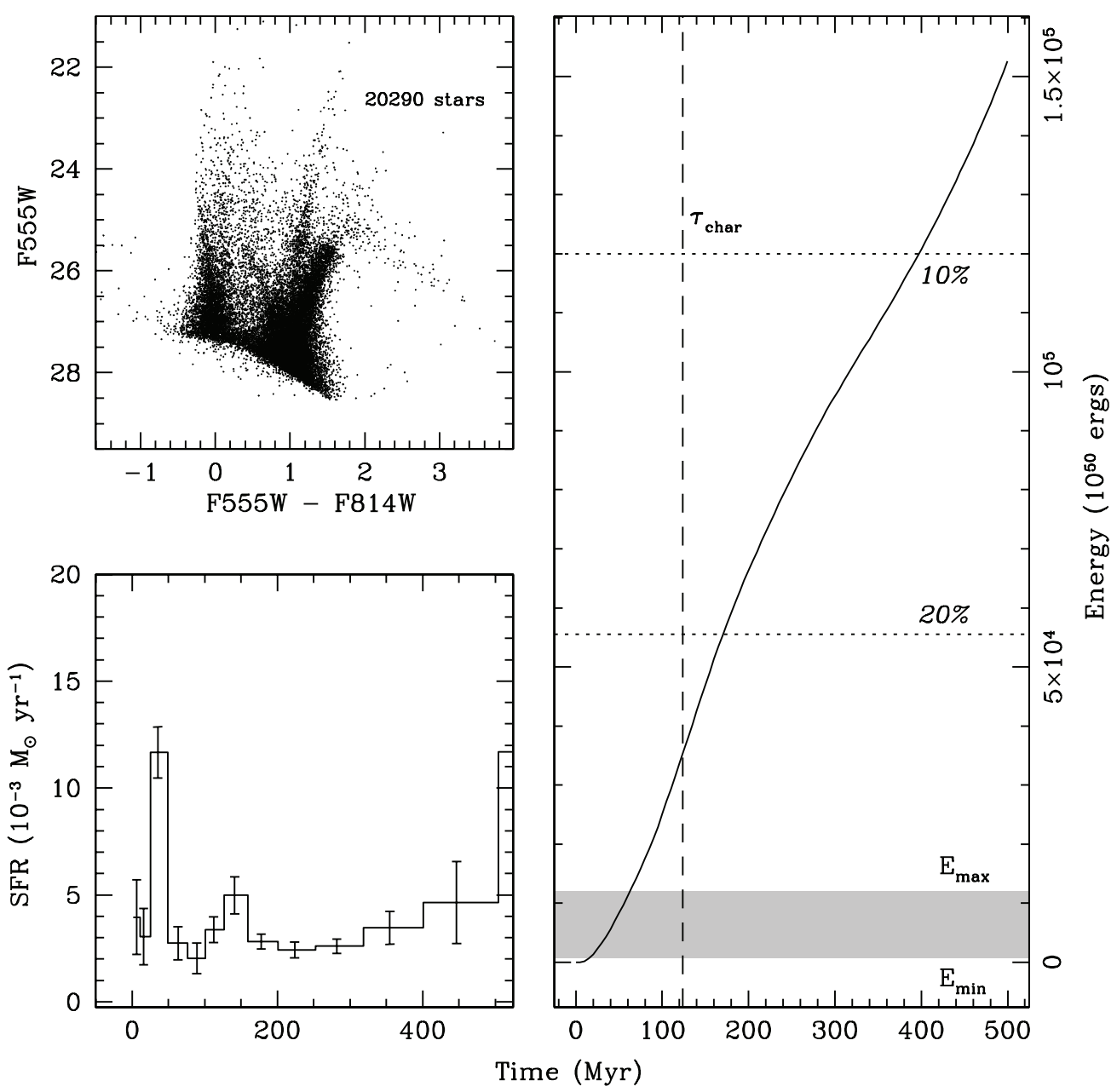

Figure 9. Same as Figure 8 except for Holmberg I.

offset from a small, higher density cloud of gas to the east. The average column density versus radius plot (Figure 7 ) gives us a hole radius of $850 \mathrm{pc}$. Figure 4 shows a blue circle denoting the boundary of the hole. This radius combined with the galaxy properties given in Tables 2 and 3 gives us a range of hole formation energies of $1.1 \times 10^{53}$ to $6.4 \times 10^{53} \mathrm{erg}$.

\subsection{5. $U G C 8508$}

First cataloged by Vorontsov-Velyaminov (1962), UGC 8508 has been studied in detail by Mould et al. (1986). These authors conclude UGC 8508 to be at a state between quiescence and bursting based upon its optical colors. UGC 8508 is relatively close by at a distance of $2.6 \mathrm{Mpc}$ (Dalcanton et al. 2009). Its $\mathrm{H}$ I angular size of roughly $110^{\prime \prime} \times 50^{\prime \prime}$ corresponds to a linear size of about $1400 \times 630$ pc. UGC 8508 has an H I integrated intensity of $13.8 \mathrm{Jy} \mathrm{km} \mathrm{s}^{-1}$ giving a total $\mathrm{HI}$ mass of $2.2 \times$ $10^{7} M_{\odot}$.

The $\mathrm{H}$ I hole is clearly observed in the southeastern part of the disk as an elliptical depression (Figure 5). We define the center of the hole to be at $\alpha=13^{\mathrm{h}} 30^{\mathrm{m}} 45^{\mathrm{s}} .9, \delta=+54^{\circ} 54^{\prime} 33^{\prime \prime}$. 0 . From the radial profile (Figure 7 ) we determine the radius of the hole to be $285 \mathrm{pc}$ leading to a hole creation energy range of $9.2 \times$ $10^{50}$ to $2.2 \times 10^{52} \mathrm{erg}$. This is the smallest and least energetic hole in our sample, yet it still occupies a large fraction of the optical extent of the galaxy (Figure 5).

\subsection{Energy Input from Stars}

To quantify the available energy from stellar processes we derive SFHs, i.e., a star formation rate as a function of time and metallicity, from the resolved stars. Color-magnitude diagrams (CMDs) contain the "fossil record" of star formation over the lifetime of a galaxy, from which we can determine an SFH. The upper left panel of Figures 8-12 show the CMDs of the stars contained within the holes. We derived SFHs by the analysis of HST-based optical CMDs using the technique described in Dolphin (2002). Briefly, this method involves the construction of single age synthetic CMDs based on a specified stellar evolution model. These individual synthetic CMDs are then linearly combined along with a model foreground CMD to produce a composite synthetic CMD. Linear weights on the individual CMDs are adjusted to obtain the best fit as measured by a Poisson maximum likelihood statistic; the weights corresponding to the best fit represent the most probable SFH.

As input, we specified a standard power law initial mass function with $x=-2.3$ from 0.1 to $100 M_{\odot}$, a binary fraction $=0.35$, the stellar evolution models of Marigo et al. (2008), distances measured from the tip of the red giant branch (Dalcanton et al. 2009), and foreground extinction values as specified by Schlegel et al. (1998). Each SFH calculation included the results from 500,000 artificial star tests, in order to simulate observational errors and completeness. For each galaxy we compared the 

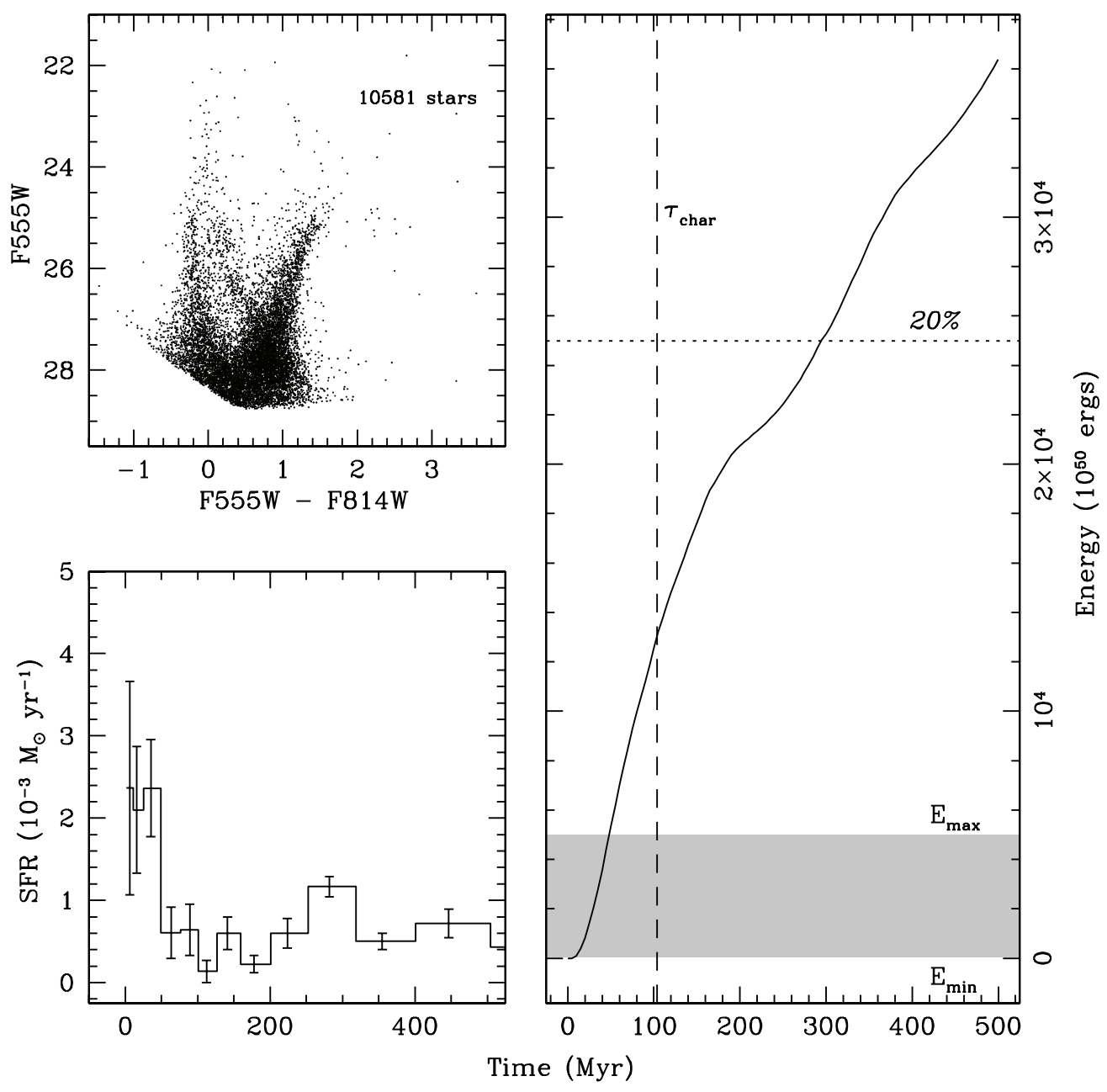

Figure 10. Same as Figure 8 except for M 81 Dwarf A.

completeness functions from the hole-only regions to the entire galaxy and found little difference. This similarity is unsurprising as our sample of galaxies does not feature significant surface brightness gradients and the holes typically cover a large portion of the HST fields. Because the CMDs considered in this analysis do not reach the ancient main-sequence turnoff, the measured chemical enrichment is uncertain (e.g., Gallart et al. 2005). We thus constrained the metallicity of each SFH to monotonically increase as a function of time, preventing potentially unphysical chemical evolution models.

To quantify the errors in the SFHs, we consider both statistical and random uncertainties. As a proxy for systematic effects, we computed the SFHs for a grid of small shifts $( \pm 0.05 \mathrm{mag})$ in distance and extinction. The rms per time bin from the grid of SFHs roughly probes systematic uncertainties in the stellar models. However, Weisz et al. (2011) show that this method may underestimate the magnitude of the systematic uncertainties by factors of a few. For the purposes of the energetics analysis in this paper, the adopted technique of deriving errors is sufficiently precise, given the larger uncertainties on $\mathrm{H}$ I energetic determinations as discussed in Section 4.2. We further computed 50 Monte Carlo realizations of the model CMD to test for effects of Poisson fluctuations on the SFH, i.e., the random uncertainties. The final error bars are simply the quadrature sum of the systematic and random errors. The lower left panel of
Figures 8-12 shows the derived SFHs over the past 500 Myr for the stars within the holes of each galaxy.

Using the above methods we produce two sets of SFHs. One set describes the global SFHs derived from all of the stars within a given galaxy. We can compare the global SFHs from the galaxies in our sample that have created large $\mathrm{H}$ i holes to SFHs of dwarf galaxies presented in the literature that have not created large $\mathrm{H}_{\mathrm{i}}$ holes. We then look for distinguishing star-forming features which may have created the observed structures. The second set of SFHs is derived using only the stars within the radius of the hole. We use these SFHs to calculate the available energy from the evolving underlying stellar population.

We next quantify the available energy from stellar evolution. The amount of stellar feedback energy produced per time bin is calculated from the galaxy evolution modeling code STARBURST99 (Leitherer et al. 1999). STARBURST99 uses stellar evolution models to estimate feedback (e.g., chemical, spectrophotometric, and stellar energy evolution) for a given SFH. We follow the method outlined in Weisz et al. (2009) in which we simulate a single burst of star formation with an initial mass of $10^{6} M_{\odot}$ and assuming a metallicity of $2 \%$ Solar, which is consistent with the expected low metallicities of these lowluminosity dwarf galaxies. We then normalize the output from the single star formation simulation by the inferred mass from the SFH, sampled in 5 Myr intervals. We plot the cumulative 

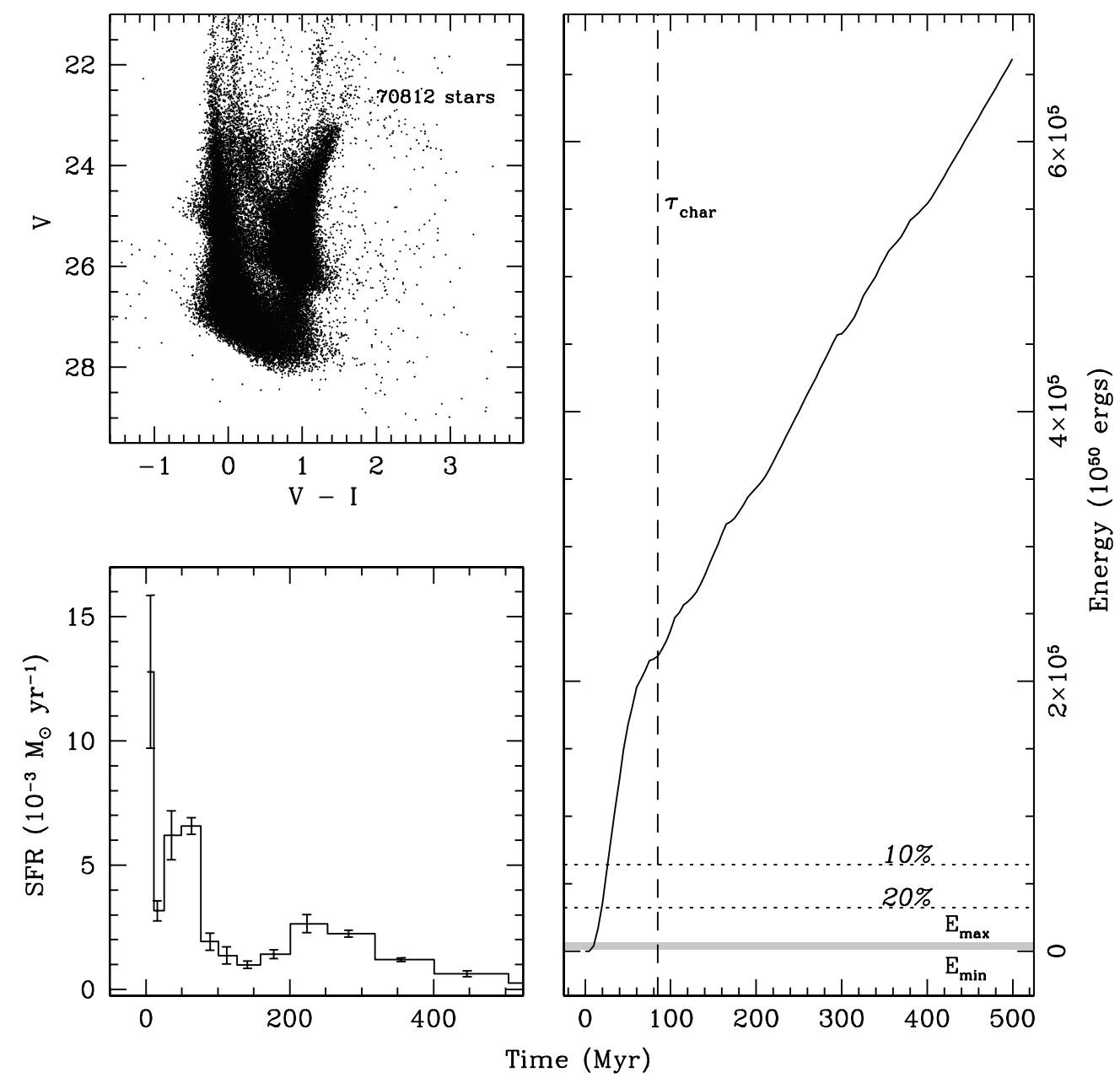

Figure 11. Same as Figure 8 except for Sextans A. The CMD is a combination of two different WFPC2 pointings of different integration times.

stellar input energies in the right panel of Figures 8-12 and discuss their implications in Section 5.

\section{COMPARISON WITH THE STELLAR ENERGY BUDGET}

We now compare the energy available from stellar feedback processes from Section 4.4 to the range of estimated hole formation energies in Table 3. The right panels of Figures 8-12 show the cumulative energy from stellar processes over the past $500 \mathrm{Myr}$. This energy profile represents a lower limit to the available stellar energy as energy estimates from STARBURST99 ignore any contribution of Type Ia SNe (contained within the hole, but created by previous generations of star formation). The vertical dashed line is the characteristic age $\left(\tau_{\text {char }}\right)$ of the hole and the shaded region is the likely range of required hole formation energies (see Section 4.2). In Table 3 we list the total amount of energy created by the stars within the characteristic age $\left(E_{\text {stars }}\right)$. In each galaxy $E_{\text {stars }} \gg E_{\min }$ implying the amount of energy available due to stellar processes within the characteristic age of the holes exceeds the minimum energies for hole formation. Using values of $E_{\min }$ implies minimum stellar feedback efficiencies $\left(\epsilon_{\min }=E_{\min } / E_{\mathrm{stars}}\right)$ of roughly $0.5 \%-4 \%$.

Alternatively, using the values for $E_{\max }$ we derive maximum formation efficiencies $\left(\epsilon_{\max }=E_{\max } / E_{\text {stars }}\right)$ between $3 \%$ and $48 \%$ (see Table 3). Note that the largest of these feedback efficiencies are above the range of $\sim 1 \%-20 \%$ inferred by models of star formation interactions with the ISM (Theis et al. 1992; Cole et al.
1994; Padoan et al. 1997; Thornton et al. 1998). However, our estimated characteristic ages are not particularly well defined. For example, Weisz et al. (2009) provide evidence that multiple generations of star formation are required to form and maintain large H I holes. Recchi \& Hensler (2006) produced a model that maintains $\mathrm{H}$ I holes for timescales as long as $\sim 600 \mathrm{Myr}$ by injecting energy from multiple generations of $\mathrm{SNe}$, prohibiting the gas from cooling and recollapsing.

With these limitations in mind we can try to determine the age of the holes in another manner. Since the models of interactions between stellar feedback processes and the ISM predict efficiencies of $1 \%-20 \%$, we can use this to determine an age for the holes assuming our upper limit for hole creation energy estimates to be correct. The dashed horizontal lines of Figures 8-12 show efficiencies of $10 \%$ and $20 \%$ using the upper limit hole creation energy estimates. For example, a $10 \%$ feedback efficiency would require the energy of 10 times $E_{\max }$ to create the hole. These lines should give us upper limits on the ages of the holes for each feedback efficiency. Clearly the inferred characteristic ages are underestimating the likely age of formation of the holes in each of the galaxies (except for UGC 8505) if energy input from multiple stellar populations have prohibited the holes from recollapsing. The inferred characteristic age of the hole in UGC 8508 is comparable to the ages determined for both $10 \%$ and $20 \%$ efficiencies, which is most likely due to the relatively small amount of required hole creation energy. 

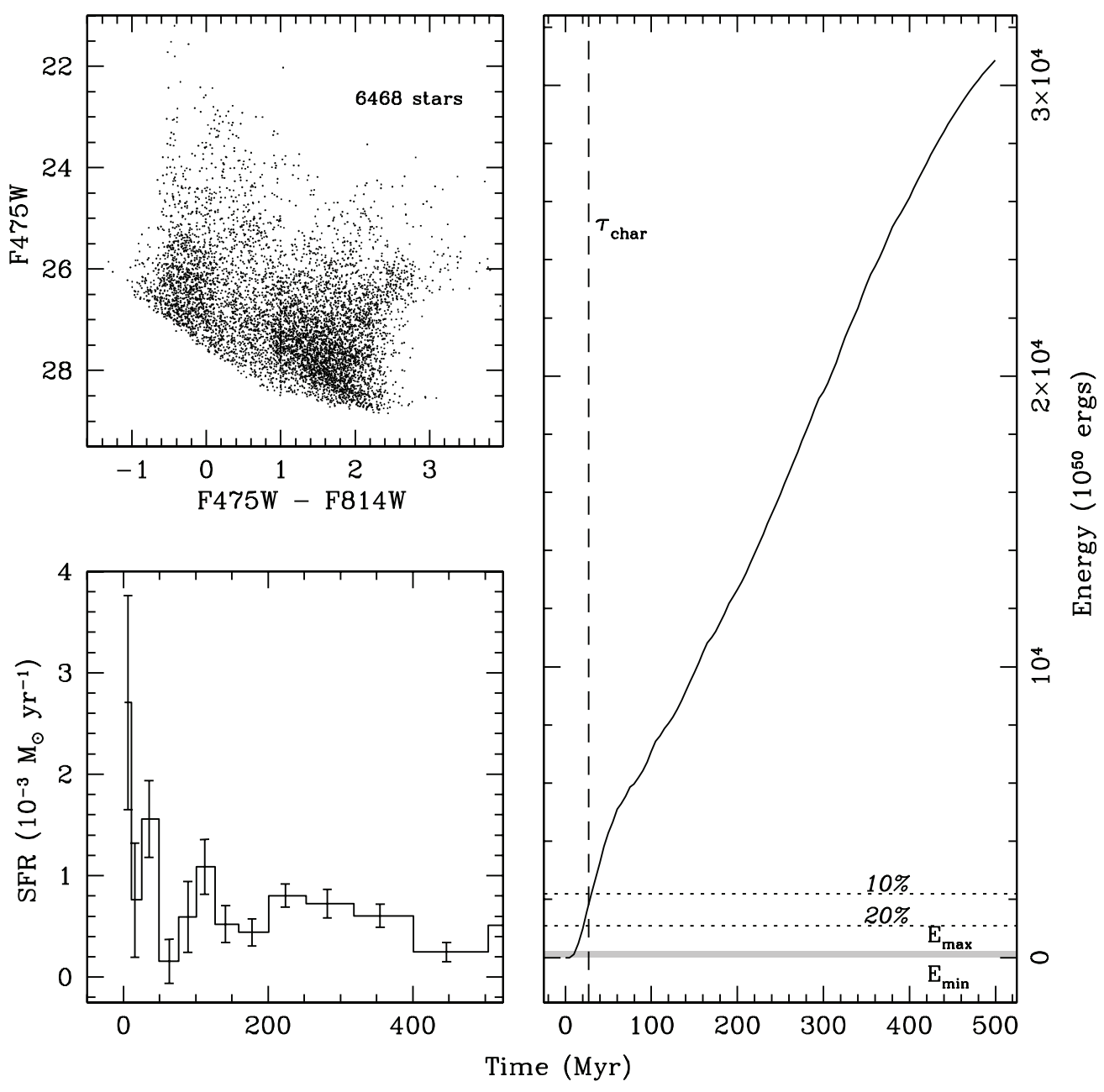

Figure 12. Same as Figure 8 except for UGC 8508.

These results illustrate the significant level of uncertainty when dealing with large $\mathrm{H}_{\mathrm{I}}$ holes that have blown out of the disk. Without the ability to accurately pin down the expansion velocity and initial distribution of the holes, estimating creation energies and hole ages are highly uncertain and perhaps even arbitrary when one assumes the standard toy model of a single burst of star formation. More likely, multiple generations of star formation work in collaboration to grow these structures to the sizes we observe today. However, even with these uncertainties, it is clear that there is no evidence yet against forming these holes through stellar feedback. Although the absolute values may vary, we have shown that the amount of energy available in the underlying stellar population is more than enough to likely be the dominant creation source.

\section{COMPARISON OF GLOBAL SFHs}

We now address the question "Is there an imprint on the global SFH of the event(s) that create the centrally dominant hole in the ISM?" To do so, we compare the recent global SFHs derived from all of the resolved stars of the five galaxies in this study to the recent global SFHs presented in Dohm-Palmer et al. (1998) and Weisz et al. (2008). We present the recent global SFHs of the five galaxies in our sample in Figure 13. There is no single feature beyond $100 \mathrm{Myr}$ ago in common when comparing the five galaxies from our large hole sample with each other. However, the most recent star formation activity
$(<100 \mathrm{Myr})$ for each galaxy in our sample shows a systematic rise that is likely due to star formation on the edges of our defined holes (see Section 7). Likewise, when we compare the SFHs in our sample with those presented in Dohm-Palmer et al. (1998) and Weisz et al. (2008) nothing obvious stands out as a major difference between the two populations. Many of the galaxies have a systematic rise in the most recent time bins whether they have created a large hole or not. Our sample contains galaxies with very low, near constant levels of star formation over the last Gyr and certainly provides no obvious recent burst that can be interpreted as a "hole creation" signature (e.g., GR8; Dohm-Palmer et al. 1998 and M81 Dwarf A). Our sample also includes galaxies that have significant star formation activity in their recent histories. However, some of these galaxies have created large, centrally dominant holes (i.e., Holmberg I), but others have not (i.e., NGC 2366; Weisz et al. 2008). This result, combined with the results from the previous section, suggests that sustained star formation is a necessary phenomenon to create large $\mathrm{H}$ i holes, but not a sufficient one.

This global comparison confirms the general results of Weisz et al. (2009). These authors looked at the many holes seen in the H I distribution of Holmberg II and compared the SFHs of the stars within the holes to those within control fields. The control fields consisted of regions of similar size but in locations where there were no Hi holes. The expectation in this previous study was to find an increased level of star formation in the regions of the holes compared to the control fields. However, the control 


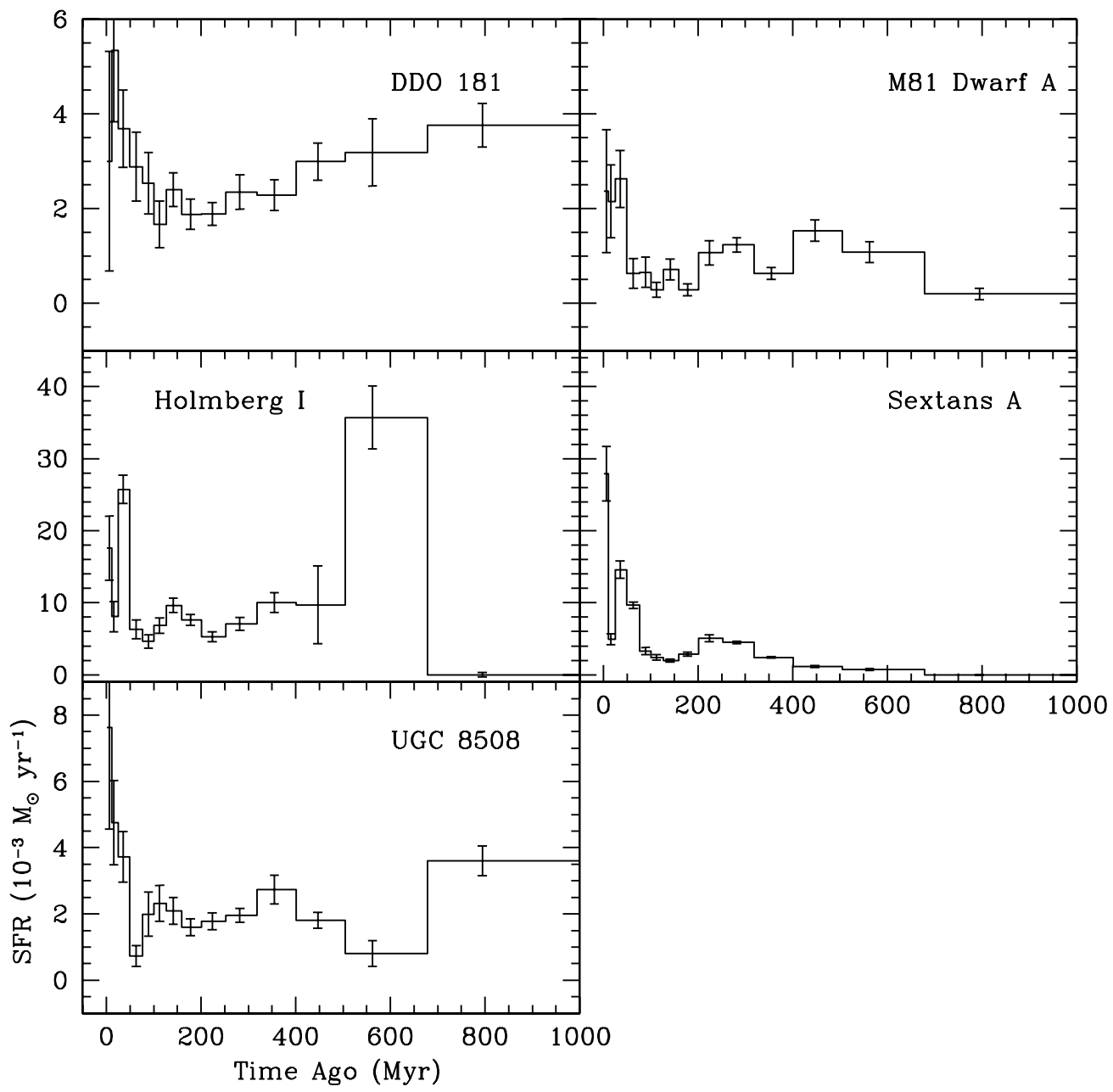

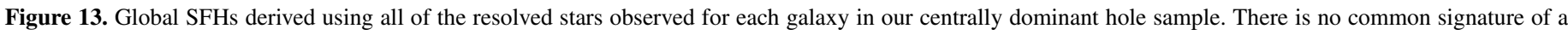

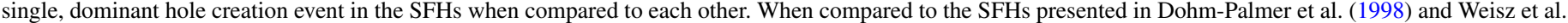
(2008), the SFHs of this study do not distinguish themselves in any way.

fields contained amounts of energy comparable to (and in some cases greater than) the areas with holes. This surprising result suggests that the local conditions of the ISM and where stars form in the galaxy play a critical factor in whether or not a hole is created. For example, perhaps the surrounding ISM must be dense enough over a large region to sustain a coherent structure prior to blow out. It is likely that the porosity of the ISM plays a very important role. A more porous medium may prohibit the formation of holes by allowing the ionized gases needed for pressure support to leak out before structures can be formed. Also, the output energy produced by star formation occurring in multiple regions within a galaxy may destructively interfere, thus destroying newly formed holes. In contrast, if star formation occurs in a consecutive and concentrated manner, the output energy may result in holes that combine to create the observed large features.

An alternative to the suggestion that these large holes are created by sustained star formation over multiple generations is that the stars play no role whatsoever and that local conditions of the ISM (e.g., gravitational instabilities) alone determine where holes are created. We do not favor this explanation since it is known that stellar processes do indeed contribute to the heating and ionization of the ISM. Our results for the large amount of available stellar energy from Section 5 and the results of the SFH comparison above seem to confirm that the stars must play a role in creating the holes, however, not at the one-to-one level. The local conditions of the ISM must also play a critical role in this process. However, the overall importance of each process is difficult to measure.

Numerical studies may be helpful to test which conditions are needed for stellar processes to create large $\mathrm{H}$ i holes. This exercise is beyond the scope of this work, but we can suggest some areas of focus. Ideally, three-dimensional magnetohydrodynamic simulations of a realistic dwarf galaxy ISM should be modeled. A parameter search should then follow including, but not necessarily limited to, simulating different total $\mathrm{H}$ I masses, varying ISM porosity values, and a range of SFHs and locations within the disk. These suggestions would expand upon the pioneering work of Fragile et al. (2003), Recchi \& Hensler (2006) and Stinson et al. (2007). Fragile et al. (2003) investigated chemical enrichment in dwarf galaxies through the simulation of supernovae in a model galaxy. Their Figures 2 and 7 show tantalizing similarities to our observed galaxies. Recchi \& Hensler (2006) showed that multiple generations of star formation can support large $\mathrm{H}$ i structures for $\sim 500 \mathrm{Myr}$ or more. Stinson et al. (2007) suggested that dwarf galaxies undergo rapid star formation in which the gas is pushed out of the galaxy core, cools, and infalls back to the core where the process is repeated in a pulsation-like "breathing mode" on 100 Myr timescales. At first glance, our findings seem to support the longer duration hole 
ages of Recchi \& Hensler (2006), and not the short-lived holes with a "breathing mode" as suggested by Stinson et al. (2007). We cannot rule out the model of Stinson et al. (2007) on longer breathing mode timescales, however. At a minimum, new models would give insight into which, if any, of these parameters plays the biggest role in the creation and sustainability of large Hi holes.

\section{SEARCHING FOR A PROGENITOR}

If the large hole in each of our galaxies were formed by a single, centralized stellar association and not via multiple stellar generations as we suggest, then observational evidence for the remnant cluster should exist. To try and identify these clusters, we use GALEX FUV and ground-based $\mathrm{H} \alpha$ imaging. Each of these wavelengths probes stellar life cycles over different timescales. UV wavelengths probe timescales up to $\sim 100 \mathrm{Myr}$ while $\mathrm{H} \alpha$ probes shorter $\sim 10$ Myr timescales. Note that these timescales are shorter than the $\sim 200$ Myr stellar dissolution timescales seen by Bastian et al. (2011). Figures 14-18 show the FUV and $\mathrm{H} \alpha$ images for each of our galaxies. We have overlaid the hole location onto each image for comparison. UGC 8508 could not be observed by GALEX due to bright foreground stars and/or too bright background levels (Lee et al. 2011) so we only show the $\mathrm{H} \alpha$ image.

The FUV and $\mathrm{H} \alpha$ images show diffuse emission within some of the holes in our sample but no bright, central feature expected for a cluster. However, each galaxy has bright features associated with recent star formation at or near the edges of our defined hole. DDO 181 has a bright FUV feature toward the southern edge of the hole that has no associated $\mathrm{H} \alpha$ emission. In Holmberg I, FUV and associated $\mathrm{H} \alpha$ emission features are located around most of the edge of the hole. The southern half of M81 Dwarf A has FUV emission, with most of it on the southeastern edge of the hole; however, M81 Dwarf A was not detected in $\mathrm{H} \alpha$. The large hole in Sextans A is filled with faint, diffuse, and point-like FUV and $\mathrm{H} \alpha$ emission. The brightest features are near the southeastern and northwestern edges of the hole. Lastly, UGC 8508 has bright $\mathrm{H} \alpha$ knots all around the edge of the defined hole. Each of the above bright FUV and $\mathrm{H} \alpha$ features are associated with the densest $\mathrm{H}$ i columns, and are not located in the center of the hole. This alone does not mean that multiple generations of stellar processes are responsible for the formation of these holes, but it does seem to rule out a young, single-aged cluster as being responsible.

Tenorio-Tagle \& Bodenheimer (1988) point out that large holes may have a stellar age gradient where the oldest stars populate the inner regions and the youngest stars fall along the edges of the hole. In this scenario of self-propagating star formation, we would expect to see knots of $\mathrm{H} \alpha$ and FUV emission at or near the edge of the observed hole where the conditions of star formation are suitable. However, we do not expect a one-to-one relationship between the hole boundaries and the $\mathrm{H} \alpha$ and FUV emission. This is due to the possibility that some of the current star formation is not associated with the large hole (i.e., stochastic star formation). Nonetheless, we indeed see hints of possible propagating star formation in some of the $\mathrm{H} \alpha$ and FUV images. These observations are consistent with the hypothesis that multiple generations of stars may provide the mechanical energy to create the large hole in each galaxy.

Sextans A is perhaps the strongest case for multiple generations of star formation creating large holes in the ISM of these galaxies. Sextans A's relatively close distance of $1.4 \mathrm{Mpc}$ allows us to resolve ISM structures on scales of $\sim 75 \mathrm{pc}$ (compared to
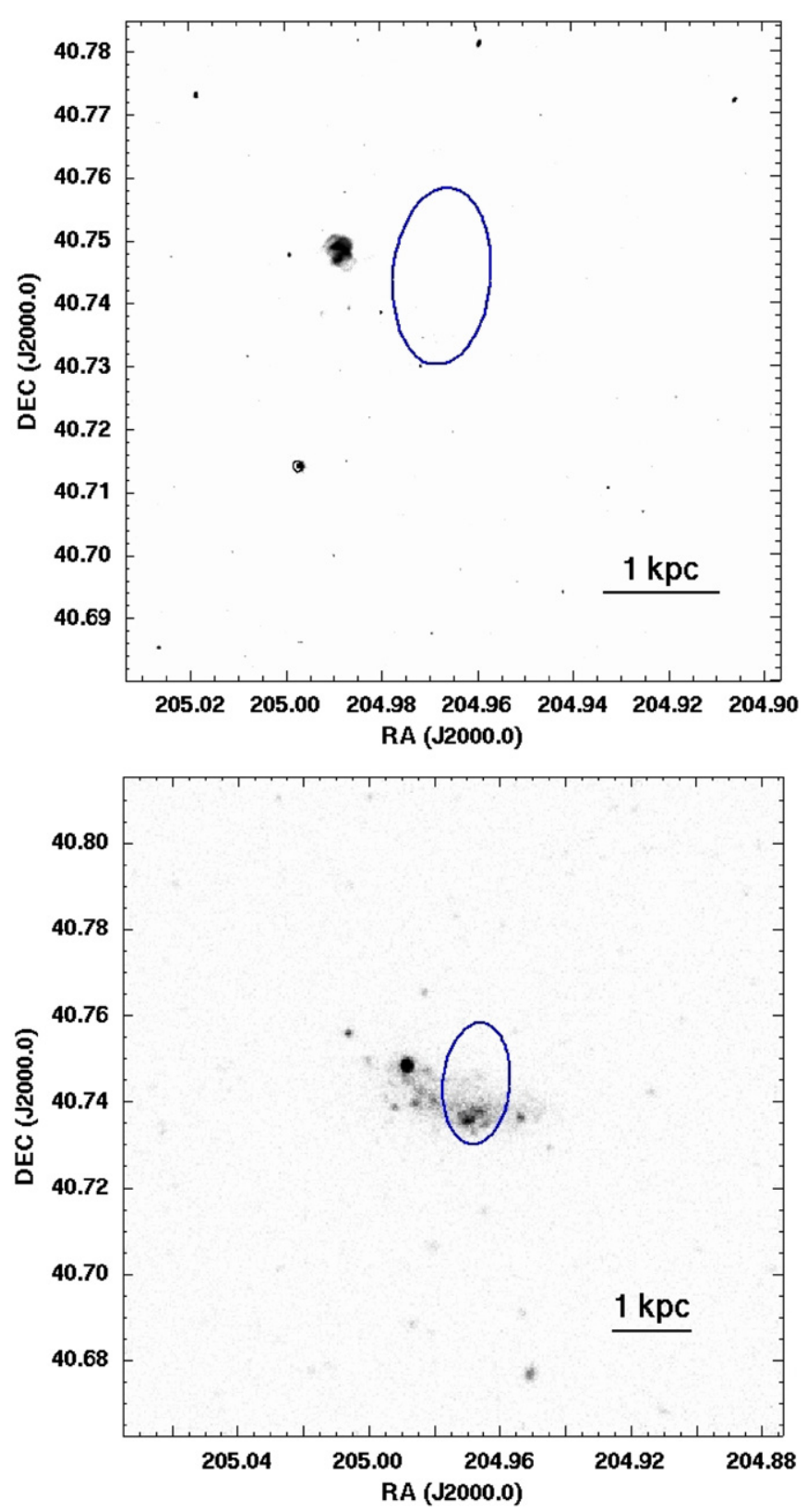

Figure 14. Ground-based $\mathrm{H} \alpha$ (top) and GALEX FUV (bottom) image of DDO 181. The blue ellipses denote the area of the hole.

(A color version of this figure is available in the online journal.)

the $\sim 300$ pc reported by Skillman et al. 1988). The central depression breaks up into multiple smaller holes when observed at our high angular resolution. Perhaps some, if not all, of the large holes in the other galaxies in our sample would also break up into multiple, smaller holes at higher angular resolution and signal to noise. Even with our incorrect assumption of a very large, single hole in Sextans A, the underlying stellar population has produced an ample amount of energy to have created a single, large hole, much less than many smaller holes. The $\mathrm{H} \alpha$ and FUV emission only cement the idea that multiple generations of star formation, not single-aged clusters, produce large $\mathrm{H}$ i holes.

\section{CONCLUSIONS}

We have investigated the genesis of large $\mathrm{H}$ i holes in the ISM of five nearby dwarf irregular galaxies by comparing the energy 

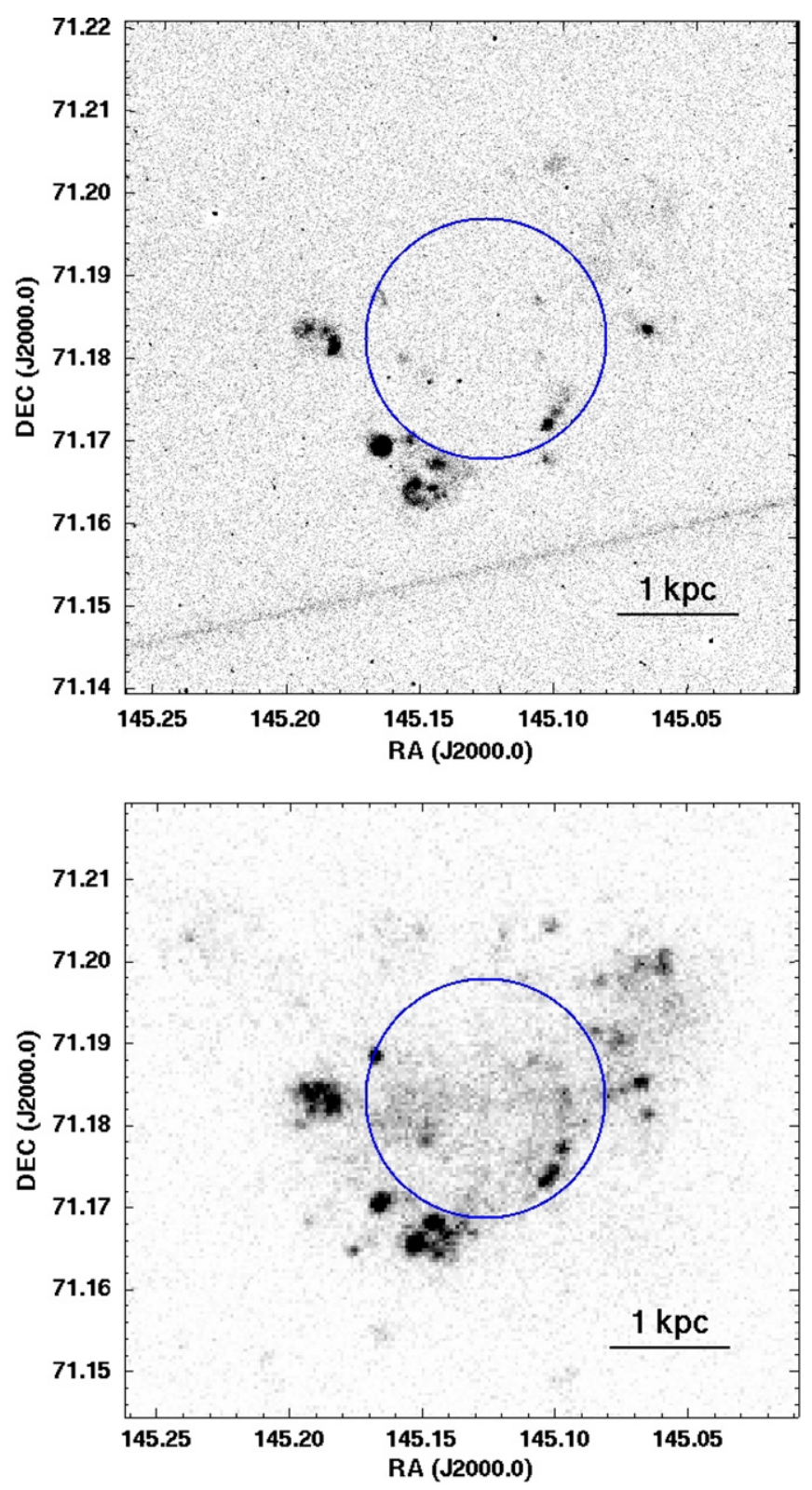

Figure 15. Ground-based $\mathrm{H} \alpha$ (top) and GALEX FUV (bottom) image of Holmberg I. The blue circles denote the area of the hole.

(A color version of this figure is available in the online journal.)

required to create the holes to the inferred input energy from the underlying stellar populations. In each galaxy, we have shown that the input energy from the stars far exceeded the required energy derived from a single blast wave model. However, based on the observations here, there is no evidence of a single star formation event associated with hole creation. Considering there is ample energy in the underlying stellar population, we conclude that multiple generations of stars are likely responsible for the creation and support of these large structures.

However, since there seems to be no correlation between the SFHs and a resultant hole, stars cannot be the only player in this game. It seems plausible that the local conditions of the ISM must also be suitable for $\mathrm{H}$ i holes to be created and sustained. Star formation has been shown to occur on the rims of holes (e.g., Weisz et al. 2009), and indeed some of the UV and $\mathrm{H} \alpha$ images of our sample galaxies also show evidence of star formation on the rim of the large $\mathrm{H}$ i holes. These new sites of star formation
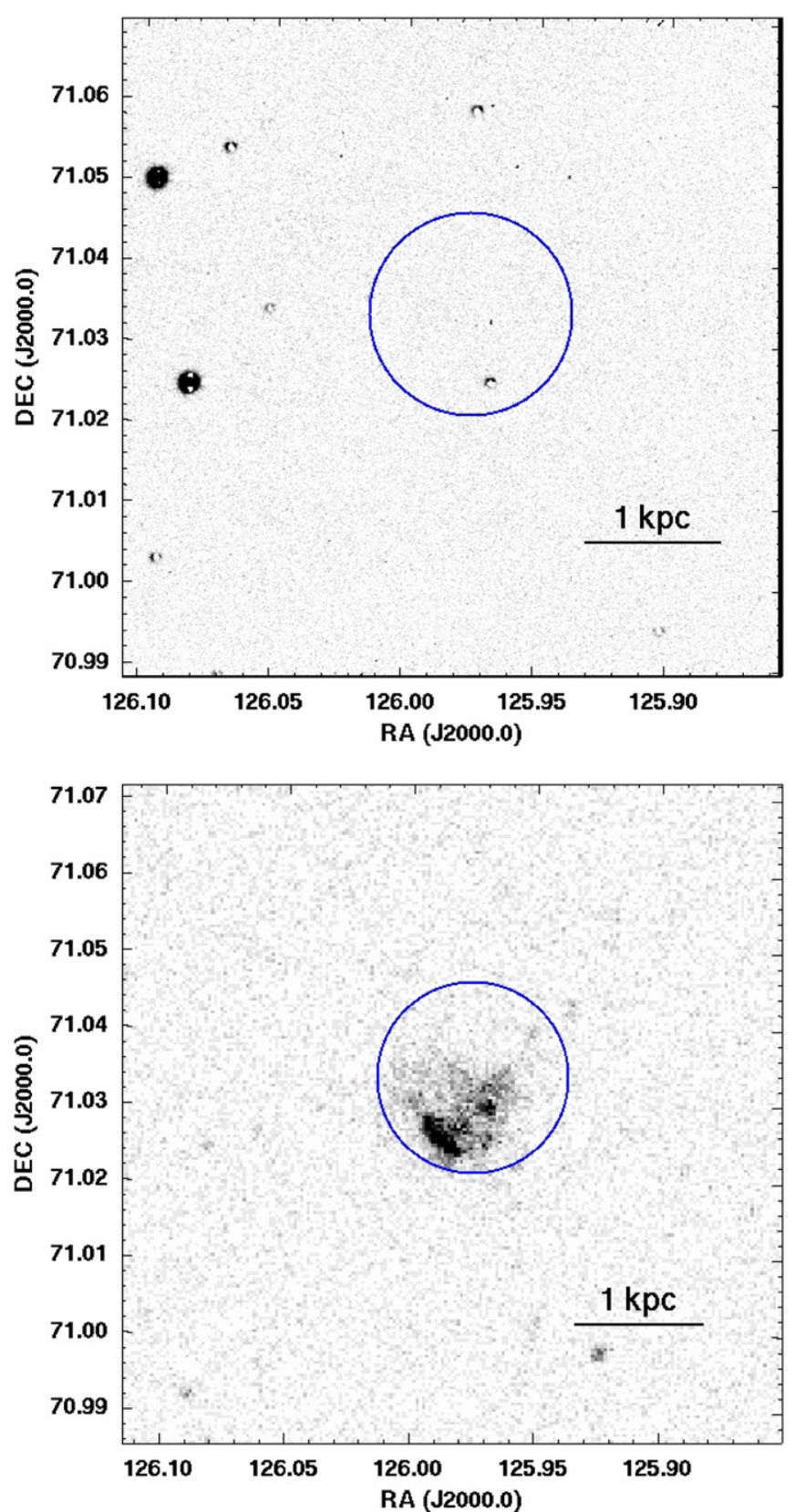

Figure 16. Ground-based $\mathrm{H} \alpha$ (top) and GALEX FUV (bottom) image of M81 Dwarf A. The blue circles denote the area of the hole.

(A color version of this figure is available in the online journal.)

can either help inflate the holes to larger sizes or destroy the holes by pushing the gas back into the cavities. It is presumably this delicate interplay between these effects that will ultimately determine whether a galaxy will or will not form a large $\mathrm{H}$ I hole.

In summary, the idea that a single-aged cluster is required to form the holes does not work based on the evidence at hand. Past numerical studies of hole creation have relied upon this tenet (see, for example, Vorobyov et al. 2004), although some numerical studies intentionally use massive, single-aged clusters to produce Type 1 holes (e.g., Mac Low \& Ferrara 1998). Future numerical studies aiming to accurately investigate hole creation should consider focusing on multiple generations of star formation occurring at different locations throughout the disk. This study reaffirms that the physics of the ISM of dwarf 

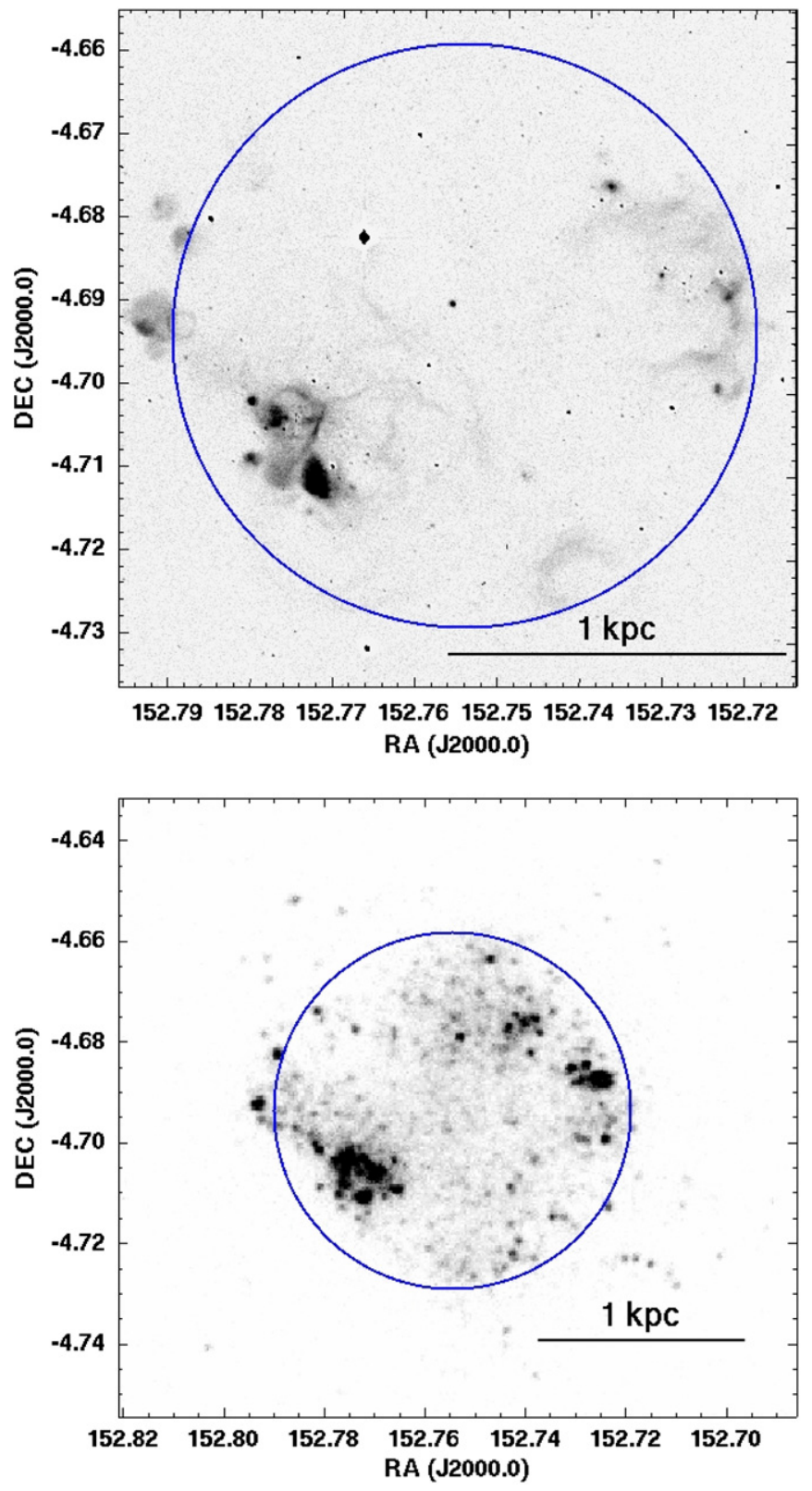

Figure 17. Ground-based $\mathrm{H} \alpha$ (top) and GALEX FUV (bottom) image of Sextans A. The blue circles denote the area of the hole.

(A color version of this figure is available in the online journal.)

irregular galaxies is complicated and one cannot use simplified approximations to predict observed distributions.

We thank the anonymous referee for a prompt and detailed report which significantly improved the clarity of the manuscript. Support for this work was provided by NRAO through the National Science Foundation collaborative research grant 807515. NRAO is operated by Associated Universities, Inc., under cooperative agreement with the National Science Foundation. S.R.W. is grateful for support from a Penrose Fellowship. This research has made use of NASA's Astrophysics Data System Bibliographic Services and the NASA/IPAC Extragalactic Database (NED), which is operated by the Jet Propulsion Laboratory, California Institute of Technology, under contract with the National Aeronautics and Space Administration.

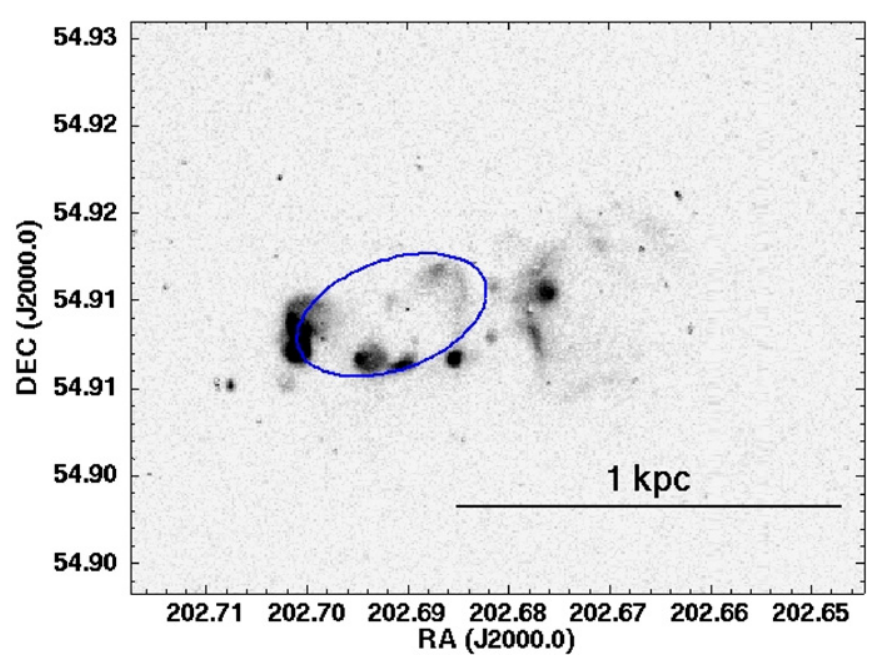

Figure 18. Ground-based $\mathrm{H} \alpha$ image of UGC 8508. The blue ellipse denotes the area of the hole.

(A color version of this figure is available in the online journal.)

\section{REFERENCES}

Bagetakos, I., Brinks, E., Walter, F., de Blok, W. J. G., Usero, A., Leroy, A. K., Rich, J. W., \& Kennicutt, R. C. 2011, AJ, 141, 23

Banerjee, A., Jog, C. J., Brinks, E., \& Bagetakos, I. 2011, MNRAS, 705

Bastian, N., et al. 2011, MNRAS, 412, 1539

Begum, A., Chengalur, J. N., Karachentsev, I. D., Sharina, M. E., \& Kaisin, S. S. 2008, MNRAS, 386, 1667

Borissova, J., Kurtev, R., Georgiev, L., \& Rosado, M. 2004, A\&A, 413, 889

Brinks, E., \& Bajaja, E. 1986, A\&A, 169, 14

Bureau, M., \& Carignan, C. 2002, AJ, 123, 1316

Cannon, J. M., et al. 2011, ApJ, 735, 36

Cash, W., Charles, P., Bowyer, S., Walter, F., Garmire, G., \& Riegler, G. 1980, ApJ, 238, L71

Chakraborti, S., \& Ray, A. 2011, ApJ, 728, 24

Chevalier, R. A. 1974, ApJ, 188, 501

Chu, Y.-H. 2008, in IAU Symp. 250, Massive Stars as Cosmic Engines, ed. F. Bresolin, P. A. Crowther, \& J. Puls (Cambridge: Cambridge Univ. Press), 341

Cole, S., Aragon-Salamanca, A., Frenk, C. S., Navarro, J. F., \& Zepf, S. E. 1994, MNRAS, 271, 781

Daigle, A., Joncas, G., Parizeau, M., \& Miville-Deschênes, M.-A. 2003, PASP, 115,662

Dalcanton, J. J., et al. 2009, ApJS, 183, 67

Dib, S., \& Burkert, A. 2005, ApJ, 630, 238

Dohm-Palmer, R. C., Skillman, E. D., Mateo, M., Saha, A., Dolphin, A., Tolstoy, E., Gallagher, J. S., \& Cole, A. A. 2002, AJ, 123, 813

Dohm-Palmer, R. C., et al. 1997, AJ, 114, 2527

Dohm-Palmer, R. C., et al. 1998, AJ, 116, 1227

Dolphin, A. E. 2000, PASP, 112, 1383

Dolphin, A. E. 2002, MNRAS, 332, 91

Efremov, Y. N., Elmegreen, B. G., \& Hodge, P. W. 1998, ApJ, 501, L163

Ehlerová, S., \& Palouš, J. 2005, A\&A, 437, 101

Elmegreen, B. G. 1997, ApJ, 477, 196

Elmegreen, B. G., Kim, S., \& Staveley-Smith, L. 2001, ApJ, 548, 749

Fragile, P. C., Murray, S. D., Anninos, P., \& Lin, D. N. C. 2003, ApJ, 590, 778

Gallart, C., Zoccali, M., \& Aparicio, A. 2005, ARA\&A, 43, 387

Gerola, H., \& Seiden, P. E. 1978, ApJ, 223, 129

Hatzidimitriou, D., Stanimirovic, S., Maragoudaki, F., Staveley-Smith, L., Dapergolas, A., \& Bratsolis, E. 2005, MNRAS, 360, 1171

Heiles, C. 1979, ApJ, 229, 533

Heiles, C. 1984, ApJS, 55, 585

Holmberg, E. 1950, Lund Medd. Astron. Obs. Ser. II, 128, 1

Holtzman, J. A., Afonso, C., \& Dolphin, A. 2006, ApJS, 166, 534

Jörsäter, S., \& van Moorsel, G. A. 1995, AJ, 110, 2037

Karachentsev, I. D., Karachentseva, V. E., Huchtmeier, W. K., \& Makarov, D. I. 2004, AJ, 127, 2031

Karachentseva, V. E. 1968, Soobshch. Byurak. Obs. Akad. Nauk. Arm. SSR, 39,61

Kennicutt, R. C., Jr., Lee, J. C., Funes, S. J., José, G., Sakai, S., \& Akiyama, S. 2008, ApJS, 178, 247 
Kim, S., Dopita, M. A., Staveley-Smith, L., \& Bessell, M. S. 1999, AJ, 118, 2797

Lee, J. C., et al. 2011, ApJS, 192, 6

Leitherer, C., et al. 1999, ApJS, 123, 3

Lo, K. Y., \& Sargent, W. L. W. 1979, ApJ, 227, 756

Loeb, A., \& Perna, R. 1998, ApJ, 503, L35

Mac Low, M.-M., \& Ferrara, A. 1998, in IAU Colloq. 166, The Local Bubble and Beyond, ed. D. Breitschwerdt, M. J. Freyberg, \& J. Truemper (Lecture Notes in Physics, Vol. 506; Berlin: Springer), 559

Mac Low, M.-M., \& Ferrara, A. 1999, ApJ, 513, 142

Marigo, P., Girardi, L., Bressan, A., Groenewegen, M. A. T., Silva, L., \& Granato, G. L. 2008, A\&A, 482, 883

Mashchenko, S. Y., Thilker, D. A., \& Braun, R. 1999, A\&A, 343, 352

McCray, R., \& Kafatos, M. 1987, ApJ, 317, 190

Morrissey, P., et al. 2007, ApJS, 173, 682

Mould, J. R., Schneider, D. P., Harding, P., \& Bothun, G. D. 1986, PASP, 98 732

Muller, E., Staveley-Smith, L., Zealey, W., \& Stanimirović, S. 2003, MNRAS, 339,105

Oey, M. S., \& Massey, P. 1995, ApJ, 452, 210

Oh, S.-H., de Blok, W. J. G., Walter, F., Brinks, E., \& Kennicutt, R. C. 2008, AJ, 136,2761

Ott, J., Skillman, E., Dalcanton, J., Walter, F., West, A., \& Koribalski, B. 2008, in ASP Conf. Ser. 395, Frontiers of Astrophysics: A Celebration of NRAO's 50th Anniversary, ed. A. H. Bridle, J. J. Condon, \& G. C. Hunt (San Francisco, CA: ASP), 376

Ott, J., Walter, F., Brinks, E., Van Dyk, S. D., Dirsch, B., \& Klein, U. 2001, AJ, 122,3070

Padoan, P., Jimenez, R., \& Jones, B. 1997, MNRAS, 285, 711

Padoan, P., Kim, S., Goodman, A., \& Staveley-Smith, L. 2001, ApJ, 555, L33

Perna, R., \& Gaensler, B. M. 2004, ApJ, 606, 326

Puche, D., \& Westpfahl, D. 1994, in Dwarf galaxies, European Southern Observatory Conference and Workshop Proceedings, Obs. de Haute-Provence, 6-9 September 1993, ed. G. Meylan \& P. Prugniel (Garching: ESO), 49, 273

Puche, D., Westpfahl, D., Brinks, E., \& Roy, J.-R. 1992, AJ, 103, 1841

Recchi, S., \& Hensler, G. 2006, A\&A, 445, L39

Relaño, M., Beckman, J. E., Daigle, O., \& Carignan, C. 2007, A\&A, 467, 1117 Rhoads, J. E. 1997, ApJ, 487, L1

Rhode, K. L., Salzer, J. J., Westpfahl, D. J., \& Radice, L. A. 1999, AJ, 118, 323

Sargent, W. L. W., Sancisi, R., \& Lo, K. Y. 1983, ApJ, 265, 711

Schlegel, D. J., Finkbeiner, D. P., \& Davis, M. 1998, ApJ, 500, 525
Silich, S., Lozinskaya, T., Moiseev, A., Podorvanuk, N., Rosado, M., Borissova, J., \& Valdez-Gutierrez, M. 2006, A\&A, 448, 123

Simpson, C. E., Hunter, D. A., \& Knezek, P. M. 2005, AJ, 129, 160

Skillman, E. D. 1996, in ASP Conf. Ser. 106, The Minnesota Lectures on Extragalactic Neutral Hydrogen, ed. Evan D. Skillman (San Francisco, CA: ASP), 208

Skillman, E. D., Bothun, G. D., Murray, M. A., \& Warmels, R. H. 1987, A\&A, 185,61

Skillman, E. D., Terlevich, R., Teuben, P. J., \& van Woerden, H. 1988, A\&A, 198, 33

Stewart, S. G., \& Walter, F. 2000, AJ, 120, 1794

Stinson, G. S., Dalcanton, J. J., Quinn, T., Kaufmann, T., \& Wadsley, J. 2007, ApJ, 667, 170

Tenorio-Tagle, G. 1981, A\&A, 94, 338

Tenorio-Tagle, G., \& Bodenheimer, P. 1988, ARA\&A, 26, 145

Theis, C., Burkert, A., \& Hensler, G. 1992, A\&A, 265, 465

Thilker, D. A., Braun, R., \& Walterbos, R. M. 1998, A\&A, 332, 429

Thornton, K., Gaudlitz, M., Janka, H.-T., \& Steinmetz, M. 1998, ApJ, 500, 95

van den Bergh, S. 1959, Publications of the David Dunlap Observatory, Vol. 2 (Toronto: Univ. Toronto Press), 147

van der Kruit, P. C. 1981, A\&A, 99, 298

van Dyk, S. D., Puche, D., \& Wong, T. 1998, AJ, 116, 2341

Vorobyov, E. I., \& Basu, S. 2005, A\&A, 431, 451

Vorobyov, E. I., Klein, U., Shchekinov, Y. A., \& Ott, J. 2004, A\&A, 413, 939

Vorobyov, E. I., \& Shchekinov, Y. A. 2004, A\&A, 416, 499

Vorontsov-Vel'yaminov, B. A., \& Arkhipova, V. P. 1962, Morphological Catalogue of Galaxies (Moscow: Moscow State Univ.), 1, 0

Wada, K., Spaans, M., \& Kim, S. 2000, ApJ, 540, 797

Walter, F., \& Brinks, E. 1999, AJ, 118, 273

Walter, F., \& Brinks, E. 2001, AJ, 121, 3026

Walter, F., Brinks, E., de Blok, W. J. G., Bigiel, F., Kennicutt, R. C., Thornley, M. D., \& Leroy, A. 2008, AJ, 136, 2563

Weaver, R., McCray, R., Castor, J., Shapiro, P., \& Moore, R. 1977, ApJ, 218, 377

Weisz, D. R., Skillman, E. D., Cannon, J. M., Dolphin, A. E., Kennicutt, R. C., Jr., Lee, J. C., \& Walter, F. 2008, ApJ, 689, 160

Weisz, D. R., Skillman, E. D., Cannon, J. M., Dolphin, A. E., Kennicutt, R. C., Lee, J., \& Walter, F. 2009, ApJ, 704, 1538

Weisz, D. R., et al. 2011, arXiv:1101.1093

Young, L. M., \& Lo, K. Y. 1997, ApJ, 490, 710 